\title{
Genome sequencing of the Trichoderma reesei QM9136 mutant identifies a truncation of the transcriptional regulator XYR1 as the cause for its cellulase-negative phenotype
}

\author{
Alexander Lichius ${ }^{1 \dagger}$, Frédérique Bidard $^{2 \dagger}$, Franziska Buchholz ${ }^{1}$, Stéphane Le Crom³ , Joel Martin ${ }^{4}$, \\ Wendy Schackwitz ${ }^{4}$, Tina Austerlitz ${ }^{1}$, Igor V Grigoriev ${ }^{4}$, Scott E Baker ${ }^{5}$, Antoine Margeot ${ }^{2}$, Bernhard Seiboth ${ }^{\text {1* }}$ \\ and Christian P Kubicek ${ }^{1}$
}

\begin{abstract}
Background: Trichoderma reesei is the main industrial source of cellulases and hemicellulases required for the hydrolysis of biomass to simple sugars, which can then be used in the production of biofuels and biorefineries. The highly productive strains in use today were generated by classical mutagenesis. As byproducts of this procedure, mutants were generated that turned out to be unable to produce cellulases. In order to identify the mutations responsible for this inability, we sequenced the genome of one of these strains, QM9136, and compared it to that of its progenitor T. reesei QM6a.

Results: In QM9136, we detected a surprisingly low number of mutagenic events in the promoter and coding regions of genes, i.e. only eight indels and six single nucleotide variants. One of these indels led to a frame-shift in the $\mathrm{Zn}_{2} \mathrm{Cys}_{6}$ transcription factor XYR1, the general regulator of cellulase and xylanase expression, and resulted in its C-terminal truncation by 140 amino acids. Retransformation of strain QM9136 with the wild-type xyr1 allele fully recovered the ability to produce cellulases, and is thus the reason for the cellulase-negative phenotype. Introduction of an engineered xyr1 allele containing the truncating point mutation into the moderate producer T. reesei QM9414 rendered this strain also cellulase-negative. The correspondingly truncated XYR1 protein was still able to enter the nucleus, but failed to be expressed over the basal constitutive level.
\end{abstract}

Conclusion: The missing 140 C-terminal amino acids of XYR1 are therefore responsible for its previously observed auto-regulation which is essential for cellulases to be expressed. Our data present a working example of the use of genome sequencing leading to a functional explanation of the QM9136 cellulase-negative phenotype.

Keywords: Single nucleotide polymorphism, SNP, Indel, Comparative genomics, Classical mutant, XYR1, Transcription factor shuttling, Cellulases, Trichoderma reesei, QM9136

\footnotetext{
* Correspondence: bernhard.seiboth@tuwien.ac.at

${ }^{\dagger}$ Equal contributors

${ }^{1}$ Research Division Biotechnology and Microbiology, Institute of Chemical

Engineering, Vienna University of Technology, A-1060 Vienna, Austria

Full list of author information is available at the end of the article
} 


\section{Background}

Public concerns about the consequences of the continued production of fuels and chemicals from fossil carbon sources have today led to intensive attempts to switch to the use of renewable carbon resources for future energy supply, such as lignocellulosic biomass from agricultural crop residues, grasses, wood and municipal solid waste. This affects not only the production of ethanol as a biofuel but also a range of key biochemical building blocks for biorefineries, including 1,4-dicarboxy acids, 3-hydroxypropionic acid, levulinic acid, and polyols; to only name a few. Thereby, the costs of cellulases and hemicellulases needed for hydrolysis of the plant biomass to soluble substrates contribute substantially to the price, and much cheaper sources of these enzymes are urgently required [1]. Consequently, there has been a renaissance in studies aimed at understanding and improving cellulase efficiency and productivity.

Trichoderma reesei (teleomorph Hypocrea jecorina), a fungus commonly found on decaying wood within a small latitude North and South of the equator, is a model fungus for cellulase and hemicellulase production [2]. Unlike other industrial fungi, mutants were all derived from a single wild-type isolate (QM6a) which was originally found during WWII on the Solomon Islands because of its ability to destroy cotton fabrics of the US Army [3]. During the first oil crisis in the 1970s, T. reesei QM6a was found being the best cellulase producer in a large screen of tropical fungi contained in the US Army Quatermaster (QM) culture collection [4]. Random mutagenesis was then used to improve this strain and establish industrial production. In the attempt to understand the mechanisms of improvement in these strains, the genomes of some strains of these mutagenesis lineages (i.e. QM9123, QM9414; NG14, RUT C30; see Figure 1) have recently been sequenced [5-8]. This led to the identification of a high number of mutated alleles, their causal involvement in enhanced cellulase production, however, still awaits verification [2].

In the course of the earliest attempts to improve $T$. reesei QM6a by mutagenesis, the mutant strain QM9136 was obtained that was unable to grow on cellulose and that did not form any cellulases on cellulose or any other inducer, such as lactose or sophorose, but otherwise displayed a normal phenotype [9-11]. The genetics underlying this cellulase-negative phenotype of this mutant are essentially unknown.

Here we report the sequencing of the genome of the cellulase-negative $T$. reesei strain QM9136. We show that - among a few other mutations that likely are irrelevant to cellulase formation - this strain contains a frameshift mutation in the major cellulase regulator gene xyr 1 [10], and that its cellulase production can be fully rescued by reintroduction of the wild-type xyr1 at its locus. We

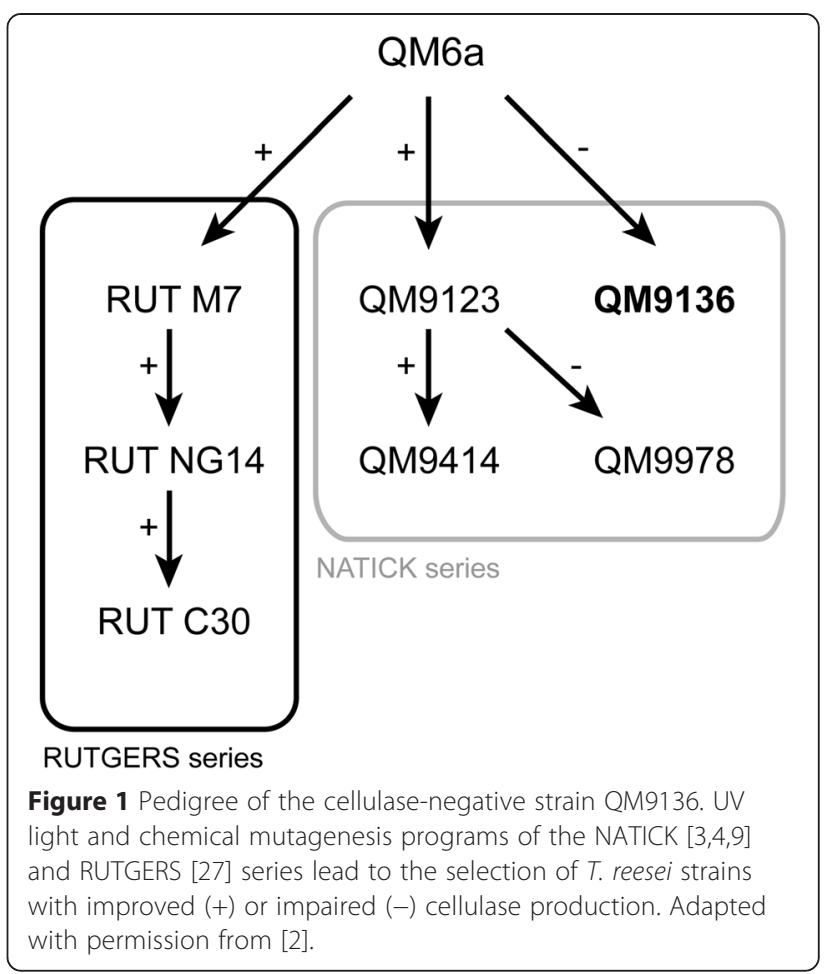

further show that this frame-shift mutation causes a 140 C-terminal amino acid truncation of XYR1, which interferes with the positive auto-regulatory feedback mechanism required for de novo XYR1 biosynthesis, efficient nuclear accumulation and transcriptional activity towards cellulase and hemicellulase gene expression.

\section{Results}

\section{Identification of mutations in QM9136}

The strain T. reesei QM9136 has been isolated by a single round of X-ray mutagenesis using a linear particle accelerator from the wild type strain QM6a [9], and was identified to be unable to produce cellulases $[9,10]$. Phenotypically, colony growth of T. reesei QM9136 on agar plates supplemented with either D-glucose, cellobiose or lactose was comparable to that of its parent T. reesei QM6a and the moderate cellulase producer QM9414 (Additional file 1: Figure S1). Interestingly, the ability of QM9136 to grow on cellulose is limited to cultivation on agar plates, and does not occur when the strain is grown in liquid culture. This phenomenon is not fully understood, but is shared with other cellulase deficient strains, including $\Delta x y r 1$ [12]. Nevertheless, in order to understand the origin of the cellulase-negative phenotype, we conducted whole genome comparisons between the natural isolate, the QM6a strain, and the mutant strain QM9136 which we sequenced using high-throughput Illumina sequencing. To get the complete picture of genetic modifications in that strain, 
this analysis included identification of the SNVs (Single Nucleotide Variants), InDels (small Insertions and Deletions) and large structural variations (deletion, duplication, translocation, insertion). The final list of mutations identified in the strain QM9136 (Table 1) has been established from in silico analysis followed by an expert analysis (see Methods). In total, 14 mutations have been detected: eight InDels, six SNVs and no structural variations. Compared to the other mutant strains sequenced [5-7], the number of mutations is much lower than that in T. reesei NG14 and RUT C30, but in the same order as in QM9123 and QM9414. This could be due to the fact that strains NG14 and RUT C30 were selected not only for cellulase hyperproduction but also for resistance against metabolic inhibitors $[5,6]$.

In order to establish the list of genes impacted by these mutations, we systematically scanned for genes in an $800 \mathrm{bp}$ window flanking each mutation. From the initial list of 14 mutations, eight genes were impacted either in their exons, their introns, or within $800 \mathrm{bp}$ upstream or $200 \mathrm{bp}$ downstream of the coding region (CDR). They were located on eight different scaffolds, and thus not clustered. Nevertheless, it was intriguing to note that they were all located either in the $5^{\prime}$ or the $3^{\prime}$ fifth of the scaffold (Additional file 2: Table S1). In addition, all mutations occurred in a genomic region that is syntenic across T. reesei, T. virens and T. atroviride (C.P. Kubicek, unpublished data).

In silico analysis of the mutations in T. reesei QM9136 identifies a truncation in the cellulase regulator XYR1.
Three SNVs were present in the CDR of the following genes: the S-adenosylmethionine synthase SAM1 (Trire2:46238); a WD-repeat containing protein that has high similarity to the cell cycle regulator CDC1 (Trire2:50707), and the subgroup B chitinase CHI18-14 (Trire2:124043; [13]; Table 1). However, in the former two proteins these SNVs only resulted in conserved amino acid changes (F13Y and S24T, respectively). The change in CHI18-14 (P389A) occurs within the C-terminal CBM1type cellulose binding domain. However, this $\mathrm{P}$ is not conserved, and is substituted by $\mathrm{G}$ or C in CBM1 orthologues from other fungi [14]. We therefore consider it unlikely that this change significantly alters the functional properties of CHI18-14.

The five indels had a more dramatic effect: in the expansin-like protein Trire2:102500 and the GT17 $\beta$ 1,4-mannosyl-glycoprotein $4-\beta-N$-acetylglucosaminyltransferase (Trire2:66687), each of the two single nucleotide losses occurred proximal to the $\mathrm{N}$-terminus, and gave rise to a completely different or truncated protein sequence, respectively (Table 1 ). As for the gene encoding the $24 \mathrm{kDa}$ subunit of the NADH-ubiquinone oxidoreductase (Trire2:5387), and a protein of unknown function (Trire2: 109416), the loss of a $T$ and a G, respectively, occurs in their promoters and the consequences of this change cannot be predicted.

\section{Consequences of the truncation on XYR1 structure}

The most interesting mutation, however, occurred in the gene encoding the transcriptional regulator XYR1

Table 1 Mutations found in T. reesei QM9136 when compared with its parent T. reesei QM6a

\begin{tabular}{|c|c|c|c|c|c|c|}
\hline SNP_id & Mutation & Mutation effect & Position* [nt] & Transcript & Element & Annotation \\
\hline 4_329479 & SNV & $G>C$ & & No & & \\
\hline 4_1496656 & SNV & $\begin{array}{l}\mathrm{A}>\mathrm{T}(\text { codon : } \mathrm{TTC}>\mathrm{TAC} ; \\
\text { aa : } \mathrm{F}>\mathrm{Y})\end{array}$ & +38 & Trire2:46238 & exon & putative S-adenosylmethionine synthetase SAM1 \\
\hline $11 \_1146347$ & SNV & $C>G$ & & No & & \\
\hline $18 \_608684$ & SNV & $\begin{array}{l}C>G(\text { codon : } A G T>A C T ; \\
\text { aa }: S>T)\end{array}$ & +71 & Trire2:50707 & exon & $\begin{array}{l}\text { putative cell-cycle regulated activator of the } \\
\text { anaphase-promoting complex/cyclosome CDC1 }\end{array}$ \\
\hline 19_523375 & SNV & $A>G$ & & No & & \\
\hline $31 \_173856$ & SNV & $\begin{array}{l}C>G(\text { codon: } C C T>G C T \text {; } \\
\text { aa : } P>A)\end{array}$ & +1189 & Trire2:124043 & exon & putative glycoside hydrolase family $18 \mathrm{CHI} 18-14$ \\
\hline $1 \_518722$ & InDel & $-1: A$ & +19 & Trire2:102500 & exon & MRSP1/expansin-like-orphan protein \\
\hline 4_1479439 & InDel & $-1: A$ & & No & & \\
\hline 5_692881 & InDel & $+1: C$ & & No & & \\
\hline 11_202303 & InDel & $-1: A$ & +2294 & Trire2:122208 & exon & transcription factor XYR1 \\
\hline 16_183301 & InDel & $-1: G$ & & Trire2:109416 & promoter & putative protein of unknown function \\
\hline 18_234489 & InDel & $-1: A$ & & No & & \\
\hline \multirow[t]{2}{*}{ 19_227418 } & InDel & $-8: G C C C G G C G$ & +19 & Trire2:66687 & exon & $\beta$-1,4-mannosyl-glycoprotein \\
\hline & & & & & & B-1,4-N-acetylglucosaminyltransferase (EC 2.4.1.144 \\
\hline 23_109645 & InDel & $-1: T$ & & Trire2:5387 & promoter & putative NADH-ubiquinone oxidoreductase \\
\hline
\end{tabular}

*in the spliced transcript. 
(Trire2:122208). The deletion of A2294 in the spliced transcript leads to a frame-shift from T2295 onwards that still encodes 16 amino acids (L765-F780: LSSTSSLRISGI PSTF) that are different to those in the native XYR1, before the first premature amber stop codon eventually terminates translation (Additional file 3: Figure S2). Consequently, $140 \mathrm{C}$-terminal amino acids are absent resulting in the truncated XYR $1^{1-780}$ protein.

A Pfam domain search [15] identified two major functional domains in XYR1: the $\mathrm{N}$-terminal $\mathrm{Zn}_{2} \mathrm{Cys}_{6}$-cluster involved in DNA binding (Gal4-like DNA-binding domain, DBD; R91-K131; $\left.1.6 \mathrm{e}^{-08}\right)$, and a fungal-specific transcription factor domain (FSTFD; R348-E701; 2.2 $\mathrm{e}^{-29}$ ). Using COILS [16], three coiled-coil regions were identified: one covering the $\mathrm{Zn}_{2} \mathrm{Cys}_{6}$-cluster and 51 amino acids $\mathrm{C}$-terminal of it (R91-L182), one at the C-terminal end of the FSTFD (S681-P712), and one at the C-terminal end of XYR1 (R890-G915) (Figure 2A). This indicates that the truncation in QM9136 starts after the FSTFD. The missing amino acids have a composition with a bias towards acidic amino acids resulting in an isoelectric point of 4 . This domain thus likely resembles the acidic activation domain (AAD) that is an invariant part of $\mathrm{Zn}_{2} \mathrm{Cys}_{6}$ transcription factors [17]. An alignment of XYR1 orthologues from several Pezizomycota shows that the missing C-terminus of XYR $1^{1-780}$ is otherwise highly conserved in all fungi (Additional file 4: Figure S3). This suggests that these 140 C-terminal amino acids are important for the function of this protein. Figure $2 B$ shows a schematic domain comparison of full-length XYR1 and truncated XYR $1^{1-780}$. Template-based protein structure modelling of this acidic activation domain showed that it forms a bundle of helices of which the last four constitute the above mentioned coiled-coil domain (Figure 2C).

The overall protein stability (estimated by computing the instability index by PROTPARAM; [18]) was determined to be 54.21 and 54.45 for XYR1 and $\mathrm{XYR} 1^{1-780}$, respectively. While this suggests that the truncation does not impact structural stability compared to the full-length protein, it should be noted that both the native and the mutant XYR1 are classified as unstable, indicating a generally high protein turn-over rate as part of XYR1's cellular function.

\section{Introduction of native $x y r 1$ into $T$. reesei QM9136 rescues the mutant growth phenotype}

The identification of a mutation in QM9136 that truncates the XYR1 cellulase regulator protein led us to assume that this may be the major or exclusive mutation that gave rise to the cellulase-negative phenotype. In order to test this, we replaced the mutated xyr1 locus in T. reesei QM9316 with a construct comprising a $2.9 \mathrm{~kb}$ wild-type copy of xyr1 N-terminally linked to $717 \mathrm{bps}$ encoding for green fluorescent protein $(g f p)$, and flanked by 1025 and 800 bps of its $5^{\prime}$ and $3^{\prime}$ UTRs, respectively. We then grew all strains on $65 \mathrm{mM}$ D-xylose as sole carbon source, which results in a compact colony phenotype in case XYR1 function is compromised. As shown in Figure 3A and B, transformation with the $g f p$-xyrl fusion gene fully restored the typical growth defective phenotype of XYR1 loss-offunction mutants on $65 \mathrm{mM}$ D-xylose [19] and matched the average colony extension speed as well as macroscopical morphology of T. reesei QM9414 and QM9414 transformed with the identical GFP-XYR1 construct (TRAL002: QM9414ttku70, Pxyr1::gfp-xyr1::Txyr1).

To test whether nuclear import of GFP-XYR1 in a QM9136 background is also sufficient to restore cellulase and hemicellulase gene expression, we performed correlated gene expression analysis on mycelia from liquid cultures induced by $1.4 \mathrm{mM}$ sophorose. The rescued QM9136 transformants indeed, expressed the major cellulase cellobiohydrolase I gene cel7a $(c b h 1)$ at levels comparable to strain QM9414 after one hour of induction with sophorose. No cellulase gene expression was observed in QM9136 or the $\Delta x y r 1$ mutant strain cultivated under identical conditions (Figure 3C). Consistent data were obtained for the xylanase II-encoding gene xyn2, hence confirming earlier data [10] that QM9136 is also deficient in hemicellulase formation (Figure 3C). While the experimental error inherent in these analyses cannot completely exclude that one of the other mutations detected (Table 1) would contribute to a very minor extent $(<5 \%)$ to the cellulase-negative phenotype, we conclude that the mutation in xyr1 has the most significant impact on QM9136's inability to produce cellulases and hemicellulases.

\section{A genetically engineered $x y r 1^{\triangle A 2294}$ allele reproduces the QM9136 mutant phenotype in T. reesei QM9414}

The above data imply that the C-terminal 140 amino acids are essential for the function of XYR1. In order to test this directly, we replaced the wild type $x y r 1$ locus in T. reesei QM9414 with the corresponding xyr1 ${ }^{\triangle A 2294}$ allele containing the truncating mutation. The resulting transformants (TRAL007: QM9414Atku70, Pxyr1:: gfp-xyr1 ${ }^{\triangle A 2294}::$ Txyrl) showed the same growth defect on D-xylose and macroscopic mutant phenotype as found in QM9136 (Figure 4A and B).

In addition, cellulase and xylanase gene expression was impaired in $T$. reesei expressing GFP-XYR1 ${ }^{\triangle \mathrm{A} 2294}$ (Figure 4C). Expression of the gene encoding cel7a after $1 \mathrm{~h}$ induction with sophorose, for instance, was 60-times lower when the truncated XYR1 version was present. Xyr1 expression, on the other hand, was only 6-times less in the mutant with truncated XYR1. We therefore conclude that presence of the genetically engineered, truncated XYR $1^{1-780}$ is sufficient to reproduce the QM9136 mutant phenotype. 


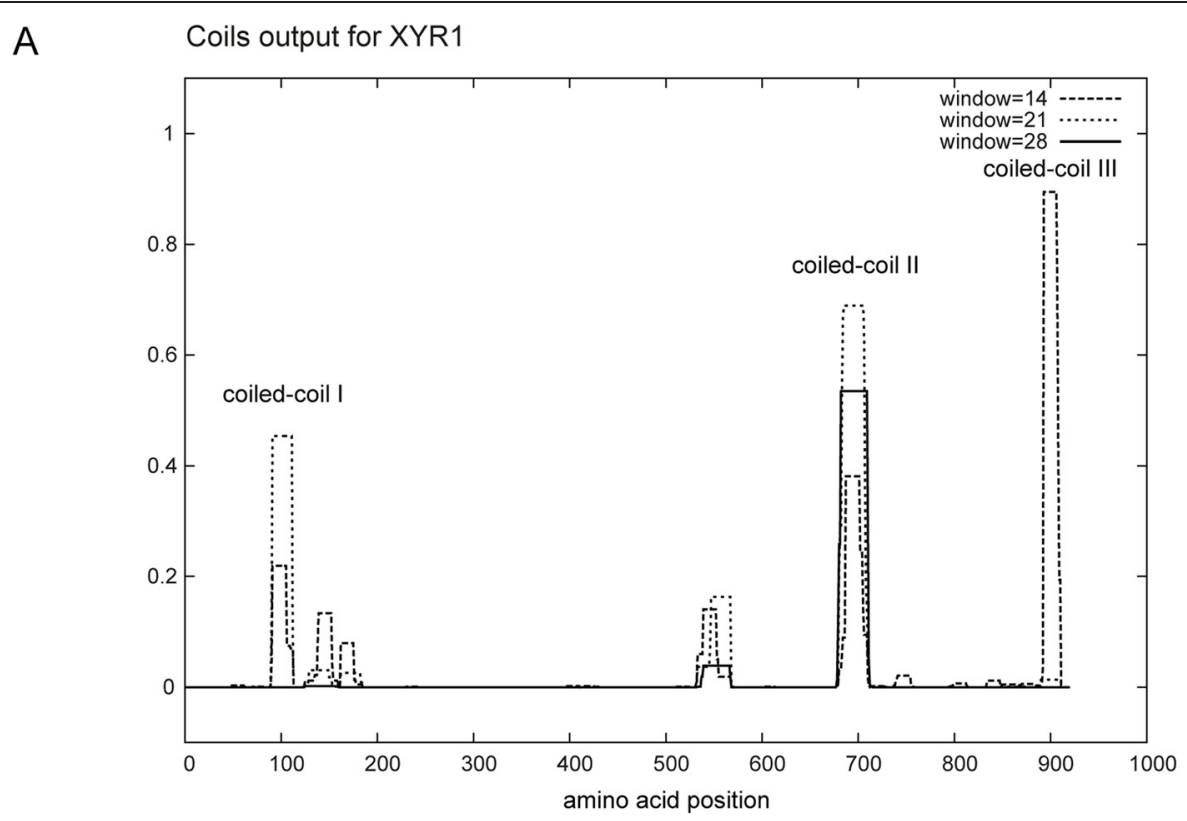

B
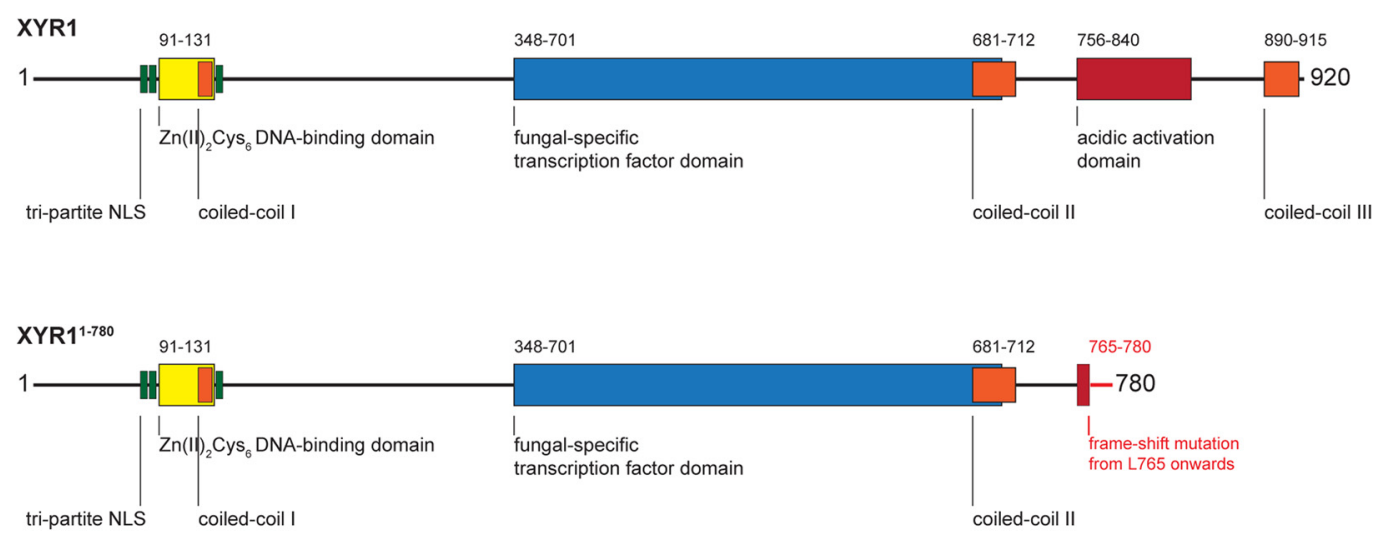

C

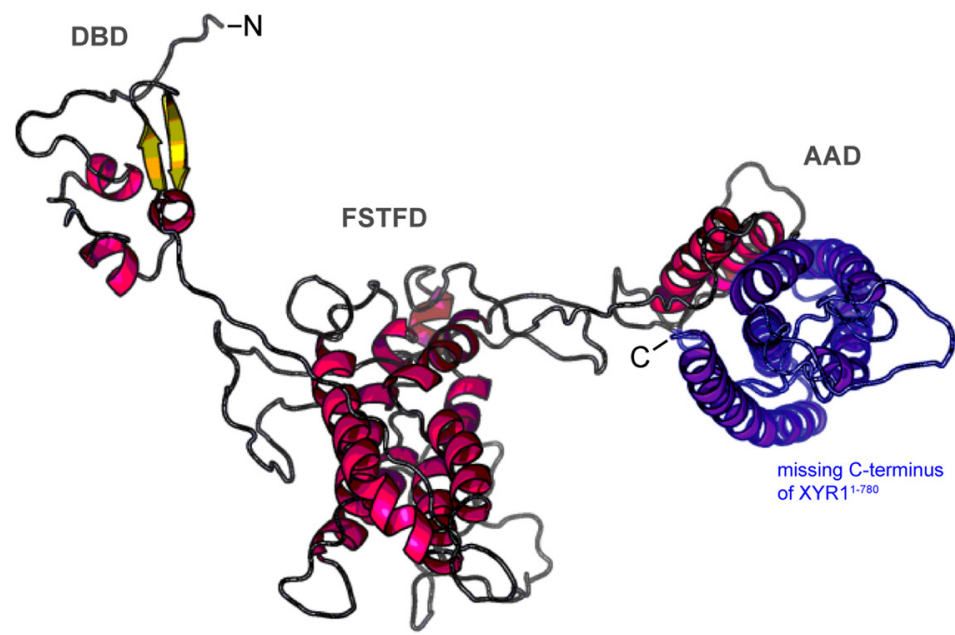

Figure $\mathbf{2}$ (See legend on next page.) 
(See figure on previous page.)

Figure 2 XYR1 structure and structural consequences of the truncated XYR ${ }^{1-780}$. (A) Predicted coiled-coil domains in XYR1 using COILS, which compares a sequence to a database of known parallel two-stranded coiled-coils and derives a similarity score from which the probability that the sequence will adopt a coiled-coil confirmation is calculated. (B) XYR1 contains a classical tri-partite nuclear localisation signal (NLS; green) which directs the transcription factor into the nucleus. XYR1's DNA-binding domain (DBD; yellow) is orthologous to that of Gal4 from Saccharomyces cerevisiae. The first coiled-coil domain of XYR1 (orange) is located at the end of the DBD. The filamentous fungal-specific transcription factor domain (FSTFD; blue) regulates target gene expression and constitutes the biggest part of the protein. A second coiled-coil domain (orange) overlaps with the C-terminal end of the FSTFD. The acidic activation domain (AAD; dark red) is a characteristic feature of $\mathrm{Zn}_{2} \mathrm{Cys}_{6}$-type transcription factors. The third coiled-coil domain (orange) sits at the very C-terminus, and likely mediates homodimerisation of XYR1. Hence, the truncated XYR1 ${ }^{1-780}$ protein encoded in QM9136 almost completely lacks the AAD and fully lacks the third coiled-coil domain due to a frame-shift mutation from L765 onwards. The terminal 16 amino acids (L765-F780; light red) encode a non-sense sequence that finds no BLASTp hit in the T. reesei genome. (C) 3D-reconstruction of XYR1. RaptorX protein structure prediction software (raptorx.uchicago.edu) identifies three functional domains in XYR1, resembling DNA-binding domain (DBD), fungal-specific transcription factor domain (FSTFD) and acidic-activation domain (AAD), perfectly matching our manual annotation (B). Five of the seven a-helices comprising the AAD are missing in the truncated XYR1 ${ }^{1-780}$ protein (highlighted in blue). The closest structural orthologs are the $\mathrm{Zn}_{2} \mathrm{Cys}_{6}$-type transcriptional regulators Ppr1 and Gal4 from S. cerevisiae, both known to be active as non-symmetrical homodimers [63].

\section{Full-length XYR1 is transported into the nucleus in $T$. reesei QM9136}

The loss of a conserved domain in a transcription factor can have three main consequences: (a) impaired stability and degradation; (b) lack of transport into the nucleus; and (c) lack of interaction with other factors responsible for enabling expression of the respective target proteins. Binding to DNA was not investigated because of the intactness of the $\mathrm{Zn}_{2} \mathrm{Cys}_{6}$ zinc finger of XYR1 in strain QM9136.

In order to test (a) and (b), we employed $\mathrm{N}$-terminal GFP-tagging of XYR1 (see above) and performed quantitative live-cell imaging analyses under cellulase inducing and non-inducing conditions. The necessity of an $\mathrm{N}$ terminal GFP fusion, and the basic nucleo-cytoplasmic shuttling dynamics of XYR1 during cellulase and xylanase expression have previously been established by us [19]. As can be seen in Figure 5A and B, the dynamics of nuclear recruitment of GFP-XYR1 in response to the presence of the inducer sophorose, were indistinguishable in QM9136 and QM9414 transformants.

Because reliable antibodies directed against XYR1 are currently not available, we furthermore took advantage of the GFP-tag to detect GFP-XYR1 by Western blot analysis, and confirmed that the high turn-over dynamics of the transcription factor previously observed in QM9414 [19], are conserved in the complemented QM9136 transformant, verifying functional rescue (Figure 6A). Summarizing, these data show that the cellular machinery required for nuclear import of XYR1 is generally intact in QM9136.

\section{The truncated XYR1 ${ }^{1-780}$ protein is also transported into the nucleus}

We consequently tested whether the mutated $x y r 1^{\triangle A 2294}$ present in QM9136 would be expressed, and - if so whether the resulting XYR $1^{1-780}$ is transported into the nucleus. To this end, we again took advantage of GFP- labelling, and studied the intracellular localization dynamics of the GFP-XYR1 ${ }^{1-780}$ construct with which we had replaced the native $x y r 1$ in T. reesei QM9414 (TRAL007).

Nuclear import of truncated GFP-XYR $1^{1-780}$ in response to an inductive signal was strongly decreased as compared to the full-length GFP-XYR1, nevertheless, still occurred at a low level. In comparison to the full-length construct which increased eight-fold within 60 minutes after replacement on sophorose, nuclear GFP-XYR $1^{1-780}$ recruitment increased only three-fold, reaching just $13 \%$ of the average GFP-XYR1 concentration (Figure 7A and B). The inability of truncated XYR1 to adequately respond to an inducing signal was observed on all tested inductive carbon sources, and indirectly correlated with the efficacy of induction (i.e. $1.4 \mathrm{mM}$ sophorose $<25 \mathrm{mM}$ lactose $<1 \mathrm{mM}$ D-xylose). Western blot analysis showed that in contrast to QM9414 and QM9136 transformants, in which full-length GFP-XYR1 (128 kDa) was readily detectable after $1 \mathrm{~h}$ of sophorose induction, only $\leq 80 \mathrm{kDa}$ large degradation products of the truncated GFP-XYR1 $1^{1-780}$ (actual size $112 \mathrm{kDa}$ ) could be detected (Figure 6B). This finding suggests that the truncated XYR1 protein undergoes proteasomal degradation much faster than the full length version, probably because it is quickly detected as non-functional.

\section{Basal XYR1 formation is unaffected by the $x y r 1^{\triangle A 2294}$ mutation}

Despite the strong reduction of the degree and rate of nuclear uptake in the strain harbouring the $x y r 1^{\triangle A 2294}$ allele, the cytosolic level of the truncated XYR1 remained low but comparable to that in the parental strain (Figure 7A). In order to rule out that this would be an artefact caused by autofluorescence of $T$. reesei, we repeated these experiments under microscope settings optimized to completely eliminate cellular background auto-fluorescence detection (see Methods). Under these stringent detection settings, any fluorescence signal must emanate from GFP only. As 


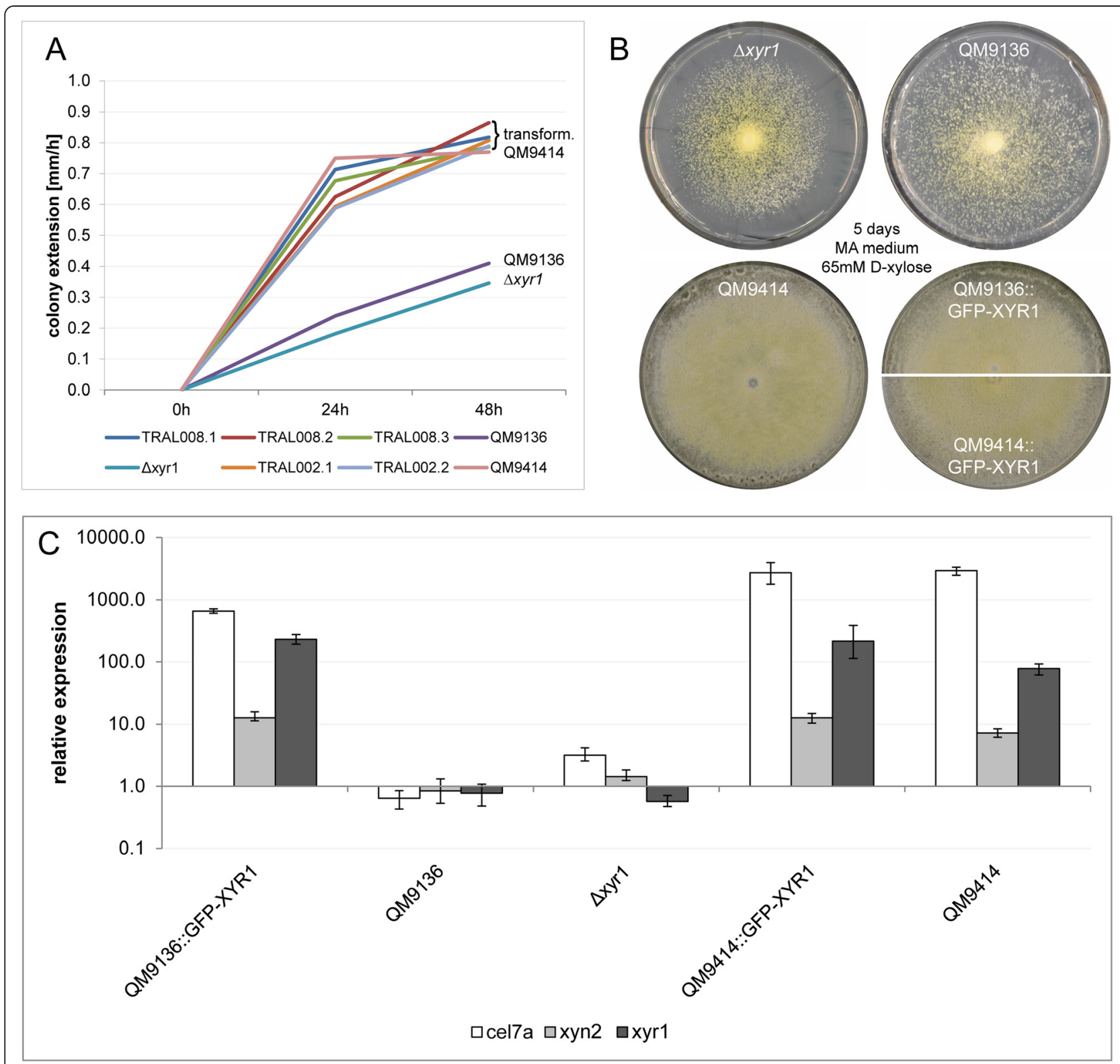

Figure 3 Gene replacement with full-length GFP-XYR1 construct rescues XYR1-loss-of-function phenotype of QM9136. (A) QM9136:GFP-XYR1 transformants (TRAL008 clones 1 to 3) grow on 65 mM D-xylose show colony extension speeds comparable to the moderate producer strain QM9414 and QM9414::GFP-XYR1 transformants (TRAL002 clones 1 and 2). QM9136 and $\triangle x y r 1$ are sensitive to 65 mM D-xylose and display significantly reduced colony extension speeds. (B) Macroscopic colony phenotypes of GFP-XYR1 transformants TRAL002 and TRAL008, and control strains after 5 days on MA medium supplemented with 65 mM D-xylose. The XYR1-loss-of-function strains $\triangle x y r 1$ and QM9136 develop a slower and less dense growing mycelium, however, start conidiation earlier, compared to the reference strain QM9414. Expression of GFP-XYR1 restores wild type morphology in QM9136 but does not alter QM9414. (C) Gene expression of the major cellulase cel7a, xylanase xyn2 and transcription factor xyr 1 itself, induced by one hour exposure to $1.4 \mathrm{mM}$ sophorose, are restored in QM9136::GFP-XYR1 transformants (TRAL008). The XYR1-loss-of-function strains QM9136 and $\triangle x y r 1$ show now transcriptional response to induction by sophorose. Both cellulase-positive controls, QM9414 and QM9414::GFP-XYR1 transformants (TRAL002) respond as expected with upregulated cel7a, xyn2 and xyr1 expression. Error bars show standard deviations ( $\mathrm{n}=6$ from three experimental and two biological replicates).

shown in Additional file 5: Figure S4, the fluorescence intensity of cytosolic GFP-XYR $1^{1-780}$ was the same under XYR1 inducing and non-inducing conditions $(1 \mathrm{mM}$ and $65 \mathrm{mM}$ D-xylose, respectively), and was also similar to that measured in $T$. reesei QM9414 expressing the fully functional GFP-XYR1. Interestingly, in this experiment we detected GFP-XYR1 $1^{1-780}$ in small cellular spots under inducing conditions, which we hypothesize might be clusters of proteasomal degradation leaving the more stable $\mathrm{N}$-terminal GFP behind. This is in line with our previously 


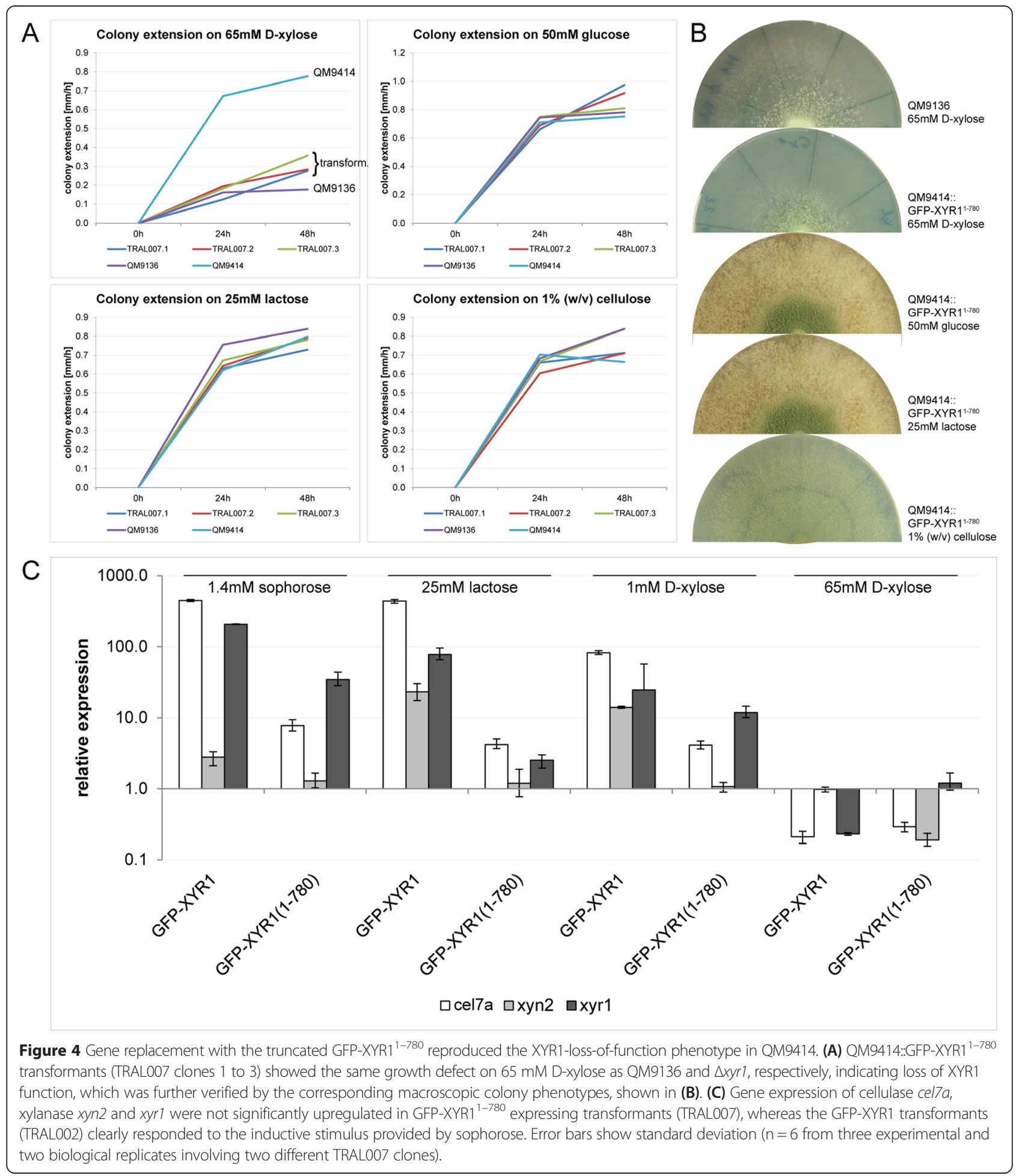

reported finding, that the GFP moiety of the fusion protein remains longer inside nuclei than the much more rapidly degraded XYR1 part [19]. Summarizing, we conclude that basal expression of $x y r 1$ is not affected by the C-terminal truncation of $X Y R 1^{1-780}$ present in T. reesei QM9136.
Impaired nuclear recruitment of XYR1 $1^{1-780}$ occurs throughout the whole fungal colony

The results documented in Figure 7 illustrate that the truncated GFP-XYR $1^{1-780}$ is indeed transported into the nucleus at the same rate as the full length GFP-XYR1, albeit the ratio of nuclear-to-cytoplasmic accumulation 


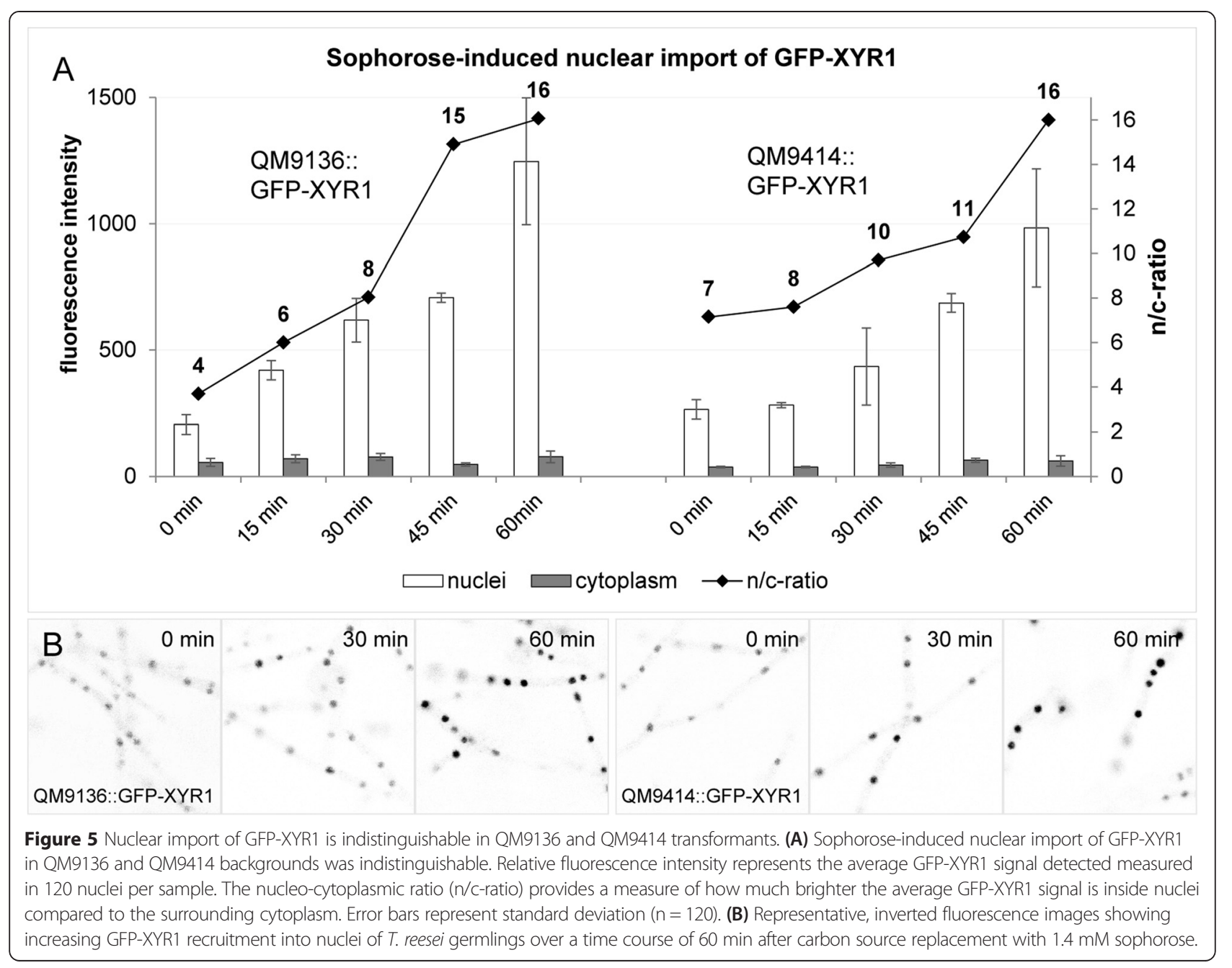

was reduced by $>75 \%$. As these experiments were conducted with germinating liquid cultures, we wondered whether this reduction in GFP-XYR $1^{1-780}$ biosynthesis and nuclear import would also occur throughout the mature fungal colony - i.e. in the colony periphery comprised of fast growing hyphae, the colony subperiphery which displays prolific hyphal branching, and the central area of the colony which is additionally characterized by aerial hyphae formation and prolific sporulation. We have recently shown that the central region of the colony was the one with the highest nuclear concentration of XYR1 [19].

Strains expressing the full-length GFP-XYR1 behaved as expected: strong nuclear recruitment of XYR1 with a clear intensity peak in the colony centre on xyrl-inducing carbon sources, and essentially the same profile but with about 10-times weaker peak intensities on xyr1-noninducing carbon sources (Figure $8 \mathrm{~A}$ and $\mathrm{B}$ ). The strain expressing the truncated GFP-XYR $1^{1-780}$ on the other hand, showed low-intensity profiles independent of the used carbon source. Notably, the overall amounts of GFP-XYR $1^{1-780}$ on any carbon source tested were comparable to those of GFP-XYR1 under non-inducing conditions, suggesting that the induction-independent baseline production of GFP-XYR $1^{1-780}$ functioned normally, however, was unresponsive to inducing signals. This was in line with data collected in shuttling experiments using conidial germlings in liquid culture. Taken together these results suggest, that C-terminal truncation of XYR1 prevents any XYR1-dependent cellular response, independent of the used carbon source, the developmental age of the fungus, i.e. germling vs. mature colony, or the functionally stratified colony region.

\section{Discussion}

Historically, the cloning of genes was based on the use of complementation of mutants, which favored those organisms which either contained plasmids or autonomously replicating elements (for easy recovering of the target gene) or which could efficiently be crossed in the laboratory. Although a number of substitute strategies had been developed (cf. [20]), this nevertheless strongly limited the progress in organisms that exhibited neither 


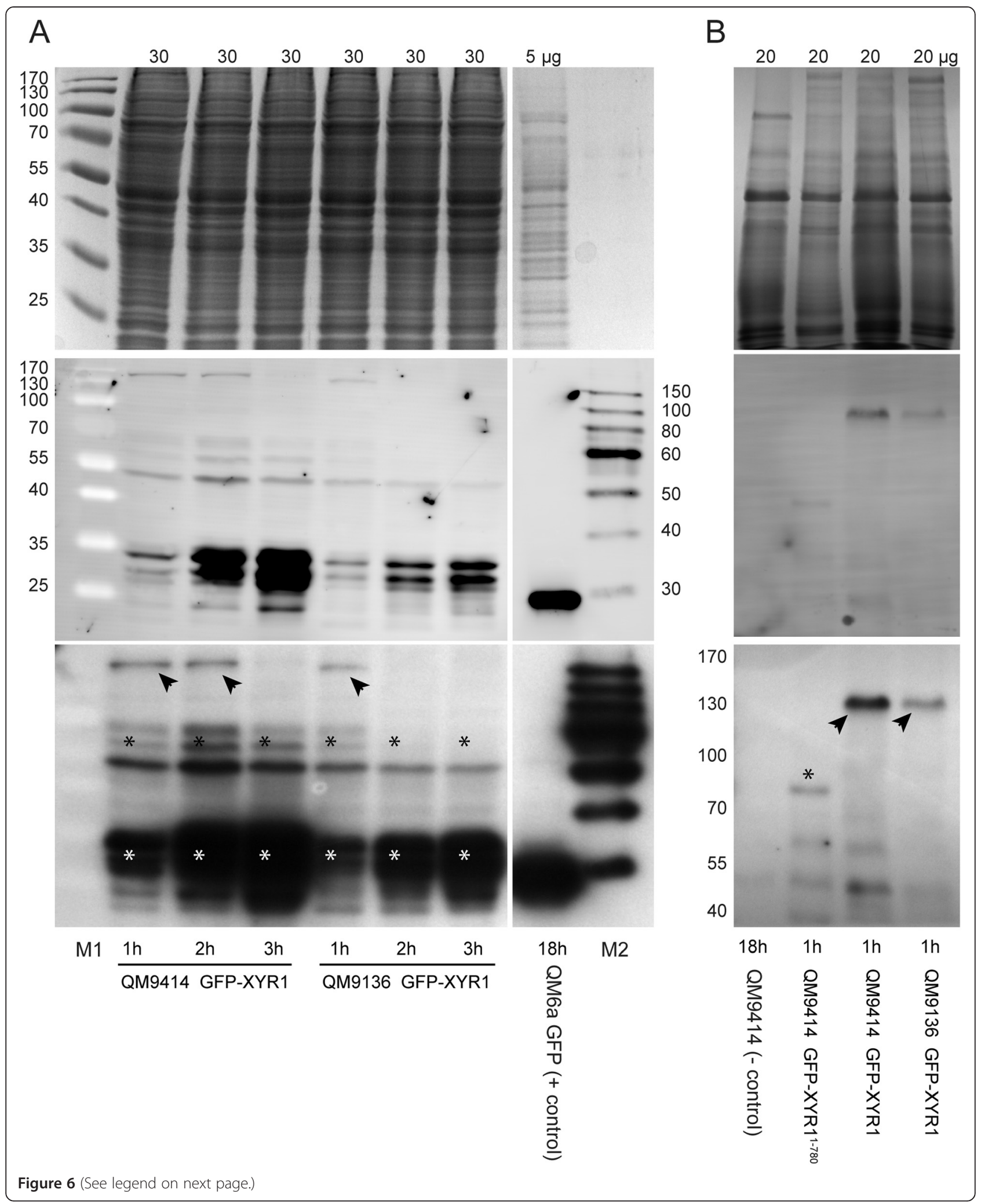


(See figure on previous page.)

Figure 6 Western blot analysis of GFP-XYR1 and GFP-XYR1 ${ }^{1-780}$ under cellulase inducing conditions. Top row: Coomassie stained loading control; middle row: chemifluorescent image of Western blot membrane; bottom row: $\mathrm{x}$-ray image of Western blot membrane. (A) With time (1-3 h of sophorose induction), less of the $127 \mathrm{kDa}$ full-length GFP-XYR1 (arrowheads) but more of its various 30-70 kDa degradation products (asterisks) can be detected in protein extracts. This rapid turn-over of the transcription factor is conserved in QM9414 and QM9136 transformants. (B) In comparison to full-length GFP-XYR1 (arrowheads) expressed in QM9414 and QM9136 transformants, of the truncated GFP-XYR1 ${ }^{1-780}$ construct, only $\sim 80 \mathrm{kDa}$ or smaller degradation products could be detected (asterisk). 5-30 $\mathrm{\mu g}$ refer to the total protein load per lane; numbers on the side denote molecular weight ranges; M1 is PageRuler pre-stained molecular weight marker; M2 is SuperSignal enhanced protein ladder; $18 \mathrm{~h}$ QM6a GFP is the positive control, and $18 \mathrm{~h}$ QM9414 the negative control for aGFP(B-2) antibody specificity.

of these possibilities, such as the so-called imperfect fungi. The advances in sequencing technologies and bioinformatics have now made it possible to sequence even full eukaryotic genomes quickly, and to use this strategy to identify mutations responsible for a particular phenotype
[21-26]. However, since mutant organisms often contain several mutations (cf. [5]), most researchers either mapped the target mutation or backcrossed their mutant against the wild type, and sequenced more than one mutant genome simultaneously.

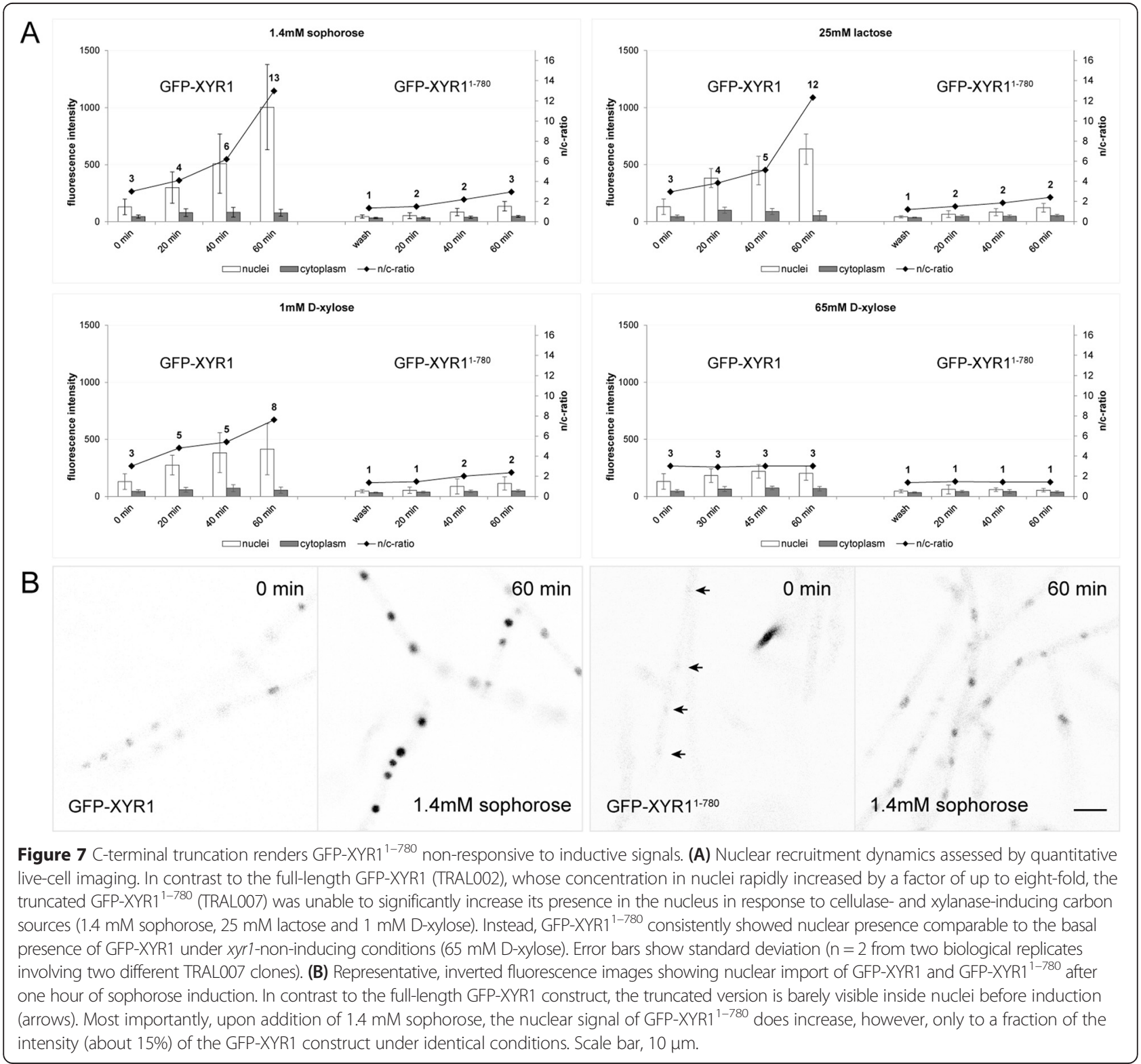


A Nuclear recruitmet of full-length and truncated XYR1 in the mature colony
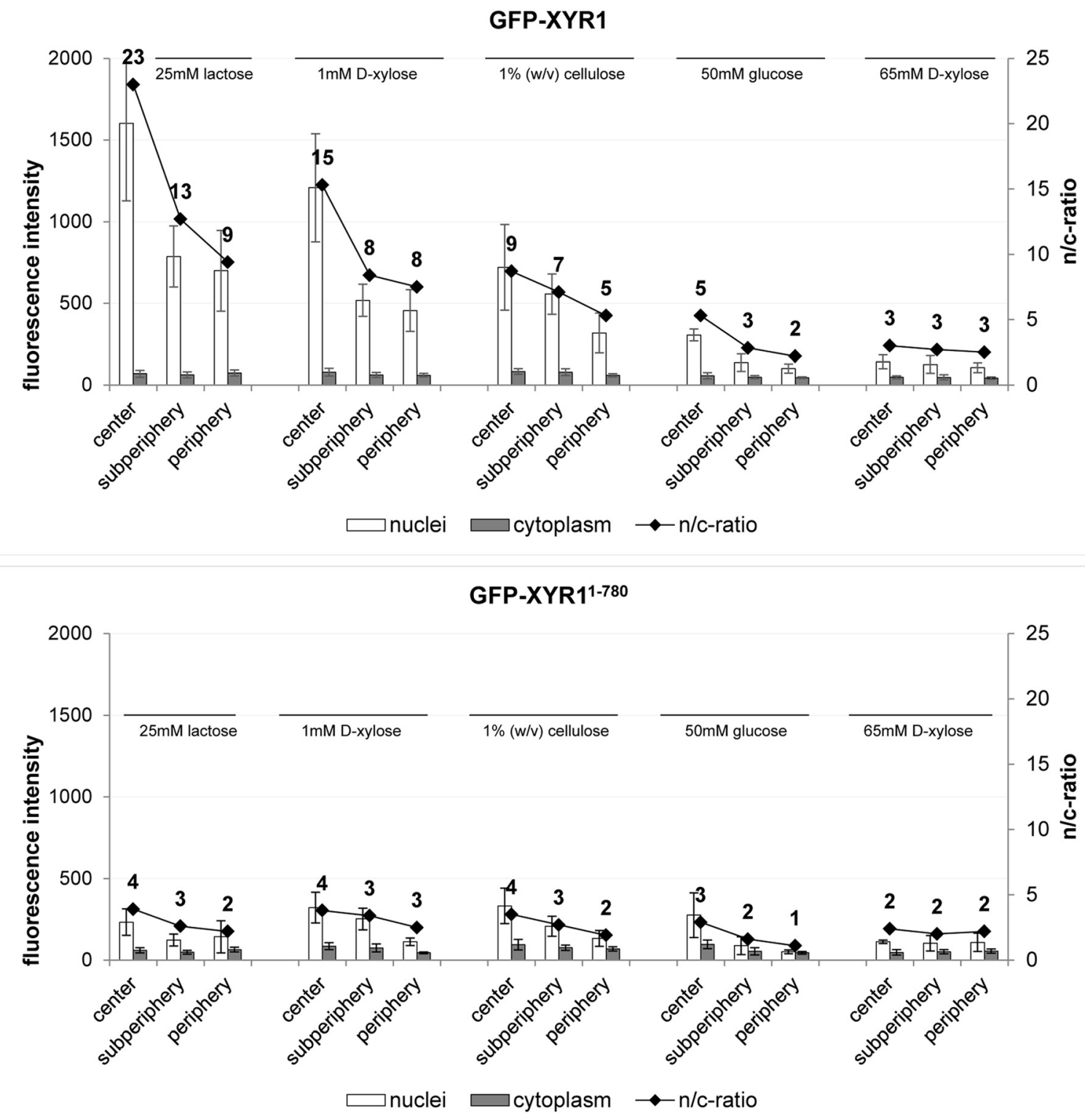

B
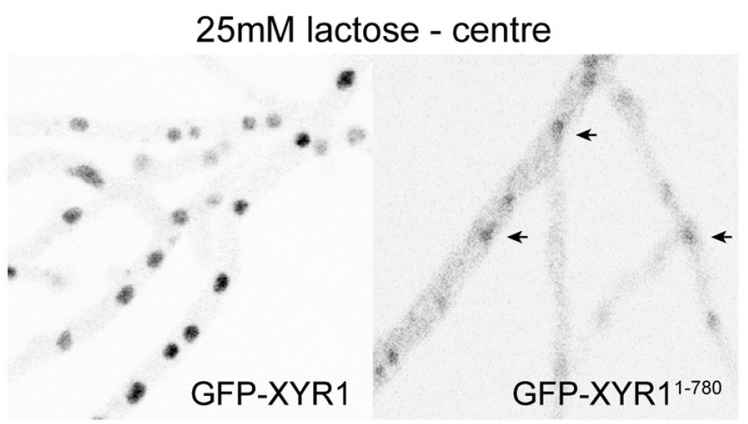

$50 \mathrm{mM}$ glucose - centre

Figure 8 (See legend on next page.) 
(See figure on previous page.)

Figure 8 Quantification of nuclear recruitment of GFP-XYR1 and GFP-XYR1 $1^{1-780}$ in the three main functional zones of the mature colony. All strains were grown for 48 hours on MA medium agar plates supplemented with the indicated carbon source. (A) Full-length GFP-XYR1 responds to different xyr1-inducing signals with nuclear recruitment, most prominently in the central area of the colony, and dependent on the inductive strength of the provided carbon source $(25 \mathrm{mM}$ lactose $>1 \mathrm{mM}$ D-xylose $>1 \%(\mathrm{w} / \mathrm{v})$ cellulose). Under non-inducing/repressing conditions (50 mM D-glucose or 65 mM D-xylose) only weak baseline nuclear recruitment can be detected. In contrast, the truncated GFP-XYR1 $1^{1-780}$ does not significantly respond to cellulase induction, and hence can only be detected at baseline levels independent of the provided carbon source. (B) Representative, inverted fluorescence images of the tested conditions, showing that GFP-XYR1 ${ }^{1-780}$ does respond to $25 \mathrm{mM}$ lactose with weak but detectable nuclear import, comparable to the baseline presence of GFP-XYR1 under repressing conditions (barely visible nuclei are indicated with arrows). On 50 mM D-glucose GFP-XYR1 ${ }^{1-780}$ is visible in small spots, suggesting degradation of the non-functional XYR1 construct in the proteasome.

Since the T. reesei mutants were maintained asexually, neither of these two strategies was possible for the present study. Hence, we were fortunate that the cellulasenegative strain QM9136 only exhibited eight mutations, of which several could be characterized as unlikely candidates for this phenotype. In accordance with the fact that T. reesei QM9136 arose from X-ray treatment (linear accelerator), which predominantly gives rise to elimination of nucleotides, we found a higher number of indels than SNVs. Vitikainen et al. [6] made the same observation for the T. reesei strains QM9414 and QM9123, which arose also by X-ray treatment $[3,9]$, whereas the $T$. reesei NG14 and RUT C30, which were isolated by UV light-treatment [27], exhibited a higher number of SNVs $[5,6]$. We found no bias in the occurrence of the mutations in the genome, as all eight occurred on different scaffolds. Yet it was intriguing to note that all but one were located at the ends of the scaffolds, and five of them occurred at scaffolds of $<1$ Mbp size. Since the size of the scaffolds is determined by the presence of long and non-alignable nucleotide repeat regions, it is possible that the proximity to regions of genomic instability facilitates the susceptibility to mutation by X-rays.

Using gene complementation, we showed that a truncation in XYR1, caused by a single nucleotide deletion, is very likely the exclusive reason for the cellulasenegative phenotype of T. reesei QM9136. XYR1 is the main actor among at least four positive transcriptional activators (XYR1, ACE2, ACE3 and the HAP2/3/5 complex), because its knock-out not only eliminates cellulases but also xylanase and $ß$-mannanase formation (for review see [28-30]). This finding is also nicely reflected in the phenotype of QM9136, which fails to grow on $65 \mathrm{mM}$ D-xylose (on agar plates and in liquid culture), has strongly reduced or no growth on lactose and cellulose, respectively, (both only in liquid culture) [31], and is deficient in the formation of cellulases, xylanases and mannanases [10].

While the identification of a gene (xyr1) that is already known to be crucially involved in cellulase and hemicellulase formation, as the cause for the inability of cellulase formation of T. reesei QM9136, may be considered trivial, it nevertheless sheds new light into its molecular function: XYR1 belongs to the fungal-specific $\mathrm{Zn}_{2} \mathrm{Cys}_{6}$ binuclear zinc cluster transcription factor family $[32,33]$. The highly conserved domain structure of this protein family contains - besides the zinc cluster domain recognizing the nucleotide binding motif - a fungal transcription factor regulating middle homology domain (FTFRMH; [34]), and short acidic activation domains within the distal portion of the C-terminus. With regard to XYR1, these domains have not yet been clearly located [35]. In this study, we have delimited these domains and consequently presented evidence that the XYR1 truncation in $T$. reesei QM9136 exclusively removed the C-terminal acidic activation domain. This implies that this domain is essential for the activation of cellulase, xylanase and $ß$-mannanase gene expression. In fact, two mutations in the XYR1 ortholog $\mathrm{X} \operatorname{lnR}$ from A. niger (L823S and Y864D) are also located in the acidic $\mathrm{C}$-terminal region and also resulted in an impairment of xylanase gene expression [36,37]. We therefore conclude that this region is essential for binding the proteins or other compounds that mediate induction of cellulase and hemicellulase formation in T. reesei. Interestingly, the above mutations lie within the four short helices that form the C-terminal coiled-coil. In the Arabidopsis thaliana TCP8 $\mathrm{Zn}_{2} \mathrm{Cys}_{6}$-type transcription factor this coiled-coil acts both as a transactivation and self-assembly domain [38]. Since the nucleotide motif that binds XYR1 has in most cases been described to only function in vivo when present as a direct or indirect repeat [39-41], we hypothesize that XYR1 has to form a dimer. A mutation in or absence of this coiled-coil domain therefore may impair binding of XYR1, and thus cellulase and hemicellulase gene expression.

Interestingly, two mutations have been reported in the $\mathrm{N}$-terminal part of the acidic activation domain that result in the opposite, i.e. constitutive expression of xylanases in $A$. niger and T. reesei and elevated basal expression of cellulases in T. reesei only. These are V756F in XlnR (corresponding to V801 in XYR1) and A804V (based on our analysis; not A824V as stated by the authors) in XYR1 $[35,42]$. Since truncation of XYR1 in QM9136 starts before both positions, this implies that the above mutations are only functional when the whole acidic activation domain is present. One model that would explain these findings is that V801 and A804 
are binding a repressor of xylanase induction, which is antagonized by binding of the proposed activator. According to Derntl et al. [35], induction of cellulase gene expression is not altered, but their basal expression level is increased. In $T$. reesei, the cellulase genes cel7a and cel6a as well as the xylanase genes $x y n 1$ and $x y n 2$ are regulated by carbon catabolite repression mainly at the basal transcription level $[43,44]$. It would thus be tempting to speculate that V801 and A804 are necessary to bind the respective catabolite repression regulator CRE1 [45,46], whose DNA-binding sites in the above genes are in close vicinity to those for XYR1 [43,44]. However, the strain of Derntl et al. [35], in which this increase of the basal level of cellulase transcription has initially been detected, is a derivate of $T$. reesei RUT C30 which bears a nonfunctional cre1 copy [46]. The nature of the repressor binding at V801 and A804 would thus need to be identified first.

We have previously shown that xyrl is transcribed at a low level constitutively, but its expression is enhanced in the presence of one of the known cellulase or xylanase inducers [19,47]. This constitutive low concentration of XYR1 is already transported into the nucleus, and we could show here that this also occurs in the QM9136 mutant. We therefore conclude that the $\mathrm{C}$-terminal acidic domain does not contain a trigger for nuclear transport. These findings are generally in accordance with those of Hasper et al. [42] who found that truncation of a $\mathrm{C}$-terminal region of $A$. niger XlnR including the coiled-coil of the fungal transcription factor activation domain (FTFAD) leads to lower but still clearly detectable nuclear localization. However, in contrast to the truncation in XlnR, the truncated XYR1 in QM9136 still bears an intact FTFAD-terminal coiled-coil region. Our data therefore suggest that the putative regulatory C-terminus of XYR1 is not per se required for nuclear import of the transcription factor, but rather appears to be involved in its induction-dependent, positive autoregulatory feedback mechanism. This explains why the fluorescent intensity of the truncated XYR1 protein stays generally low because the protein cannot be upregulated by its own production and achieve sufficient nuclear accumulation to trigger cellulase and hemicellulase gene expression.

\section{Conclusions}

Our study demonstrates how high-throughput genomic sequencing combined with reverse genetics can provide novel functional insights into organisms that are otherwise only poorly susceptible to mutational analyses. The mutation in xyr1 in T. reesei QM9136 also allowed new insights into the function of this central cellulase regulator. The ongoing investigation of yet two more cellulasenegative strains of T. reesei (i.e. QM9978 and QM9979; [48]) - which bear a functional xyr1 copy (C.P. Kubicek, B. Seiboth, A. Margeot and S.E. Baker, unpublished data) - by a similar approach is expected to further elucidate cellulase and hemicellulase regulation in this important filamentous fungus.

\section{Methods}

\section{Strains and growth conditions}

The Trichoderma reesei strains QM6a (ATCC13631), the ex-type isolate [49] QM9136 (ATCC 26920), a mutant defective in cellulase production [9,10], QM9414 (ATCC 26921), an early cellulase producing mutant derived from isolate QM6a, and its $\Delta x y r 1$ knock-out strain [12], were used throughout this work. T. reesei transformant strains expressing green fluorescent protein (GFP)-labeled XYR1 fusion proteins were generated in a QM9414 background strain which had the ku70 gene (responsible for non-homologous recombination by end-joining DNA) removed [50]. The used parental strain (QM9414 $\Delta t k u 70)$ was constructed similar to the $\Delta k u 70$ strains as described previously [50], with the only difference that the selection marker for the targeted ku70 gene knock-out was the ptrA gene [51].

Strain propagation, transformant selection and purification were performed on potato dextrose agar (PDA) supplemented with suitable selection agent when required. For submerged cultures, strains were grown in Mandels-Andreotti (MA) medium [52], using D-glucose, D-xylose, glycerol, lactose, sophorose or cellulose as sole carbon sources at final concentrations for up to $1 \%(\mathrm{w} / \mathrm{v})$ as indicated. All strains are maintained as $50 \%(\mathrm{v} / \mathrm{v})$ glycerol stocks at $-80^{\circ} \mathrm{C}$. Table 2 lists all strains used and generated in this study.

Table $2 T$. reesei strains used and produced in this study

\begin{tabular}{|c|c|c|c|c|}
\hline Strain & Strain number & Parental strain & Genotype & Reference \\
\hline QM9414 & ATCC26921 & QM6a & - & [52] \\
\hline QM9414 $\Delta t k u 70$ & - & QM9414 & $\Delta k u 70: p t r A$ & this study \\
\hline GFP & - & QM6а & Ptef1::gfp & [19] \\
\hline$\Delta x y r 1$ & - & QM9414 & $\Delta x y r 1: . h p h$ & [12] \\
\hline GFP-XYR1 & TRAL002 & QM9414 $\Delta$ tku70 & $\Delta$ ku70:ptrA; $\Delta x y r 1:$ hph; Pxyr1::gfp-xyr1::Txyr1 & [19] \\
\hline GFP-XYR1 $1^{1-780}$ & TRAL007 & QM9414 $\Delta \mathrm{tku} 70$ & $\Delta k u 70: p t r A ; \Delta x y r 1:$ hph; Pxyr1::gfp-xyr1 ${ }^{\Delta A 2294 .: T x y r 1}$ & this study \\
\hline GFP-XYR1 & TRAL008 & QM9136 & 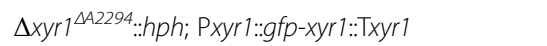 & this study \\
\hline
\end{tabular}


Minimal medium [53] supplemented with $1-2 \%(\mathrm{w} / \mathrm{v})$ sugars (D-glucose, cellobiose, lactose or cellulose) were used to determine growth phenotypes on agar plates. Plates were centrally inoculated with small agar blocks carrying mycelium, and incubate for $3-5$ days at $30^{\circ} \mathrm{C}$.

Escherichia coli strains JM109 (Promega, Madison, Wisconsin), One Shot ${ }^{\circ}$ Top10 (\#C4040-10, Life TechnologiesInvitrogen, Austria) or Stellar (\#636763,Takara Bio Europe/ Clontech, Saint-Germain-en-Laye, France) were used for plasmid construction and amplification using standard molecular cloning techniques [54].

\section{Illumina genome sequencing of $T$. reesei QM9136}

Chromosomal DNA from T. reesei QM9136 was prepared as described previously [5]. Fragment libraries were prepared according to the TruSeq ${ }^{\circ}$ DNA Sample Preparation Guide from Illumina (www.illumina.com). These libraries were then loaded onto the cluster generation station for single molecule bridge amplification using the Standard Cluster Generation kit from Illumina. The slide with amplified clusters was then subjected to sequencing on the Illumina Genome Analyser I (GAI) for single reads using the 36 cycle Sequencing Kit version 1 from Illumina.

The whole genome sequence of T. reesei QM9136 was deposited at the Sequence Read Archive (SRA; http:// www.ncbi.nlm.nih.gov/sra) under the accession number SRX059777.

\section{Sequence alignment and analyses}

Illumina short reads from QM9136 were mapped onto the T. reesei genome (http://genome.jgi-psf.org/Trire2/), using the Maq 0.6.6 software solution [55]. Mapping was done with two maximum mismatches. InDels and SNVs were also identified using Maq 0.6.6. Homozygous mutations were selected and filtered on genomic context (complexity $=1$, uniqueness $>15.8, \mathrm{GC}$ percentage between 0.31 and 0.74 ). Each mutation was manually checked using the Integrative Genome Viewer and compared to sequence of the RUT lineage (Figure 1) to remove the false mutation coming from initial QM6a error sequencing. Large structural variations were searched by using the BreakDancer software [56] and filtered on the reads number covering the genomic variations.

We evaluated the location of SNVs and deletions according to gene annotations using the "filtered models" from the JGI website. From this annotation we calculated the position of intron, promoter (using an $800 \mathrm{bp}$ upstream region) and terminator (using a $200 \mathrm{bp}$ downstream region).

\section{Generation of gene replacement cassettes}

To express GFP-labeled versions of full-length and truncated XYR1 transcription factor from its native locus, gene replacements cassettes were constructed that exchanged the native $x y r 1$ open reading frame with full length or genetically truncated copies of each $g f p$-fusion gene and the hygromycin resistance expression cassette marker gene ([57]; Additional file 6: Figure S5). C-terminal truncation of XYR1 was achieved by reproducing the deletion of A2294 ( $\triangle \mathrm{A} 2294)$ identified in the QM9136 genome through side-directed mutagenesis in the xyr1 locus of the replacement cassette, leading to the expression of a GFP-XYR $1^{1-780}$ construct in the resulting transformants. Homologous recombination was facilitated via the native $\sim 1 \mathrm{~kb} 5^{\prime}$ and $1 \mathrm{~kb} 3^{\prime}$ flanking regions. Assembly of the individually generated amplicons from $T$. reesei genomic DNA was performed using InFusion ${ }^{\circ}$ recombinational PCR cloning (HD \#639649, Takara Bio Europe/Clontech, Saint-Germainen-Laye, France). In-frame cloning of all four gene replacement cassettes was verified by nucleotide sequencing of PCR-amplicons generated from extracted, genomic DNA of the respective transformants.

\section{$T$. reesei transformation and genotyping by PCR and sequencing}

Gene replacements cassettes were amplified from the respective plasmids by PCR and transformed into T. reesei QM9414 $\Delta t k u 70$ as linear DNA fragments using electroporation as described previously [58]. Transformant strains were selected on PDA hygromycin medium $(100 \mu \mathrm{g} / \mathrm{ml}$ hygromycin final concentration), and homokaryons were obtained by repeated rounds of vegetative spore propagation on selective medium. Individual isolates were genotyped for integration of the replacement cassette at the target gene locus by PCR as described in detail elsewhere [59]. Briefly, primer pairs, binding inside and outside of the replacement cassette and native ORF, respectively, were used to probe for: (1) the integration of the replacement cassette at the target gene locus, (2) the presence of the $h p h$-resistance marker cassette at this locus, (3) the presence of GFP at this locus, and (4) the absence of the target ORF from the whole genome. When reactions were performed as multiplex PCRs, an additional pair of oligonucleotides binding within the actin locus (Trire2:44504) was used as an internal positive control for each reaction. Selected amplicons resulting from PCR genotyping were furthermore subjected to DNA sequencing, notably for verification of the $x y r 1^{\triangle A 2294}$ point deletion in TRAL007 transformants. Table 3 lists all oligonucleotides used for PCR genotyping and DNA sequencing in this study.

\section{Real-time monitoring of XYR1 nuclear transport by quantitative live-cell imaging}

In order to monitor rapid changes in the subcellular localization of GFP-tagged XYR1, carbon source 
Table 3 Oligonucleotides used in this study for PCR genotyping and DNA sequencing

\begin{tabular}{ll}
\hline Primer name & $\mathbf{5}^{\prime}$-3' sequence \\
\hline PCR genotyping & \\
Pxyr1-ver-F & AAGGATGCCGACTTAACGAAC \\
Txyr1-ver-R & AGTCGCTCATGATCCTACCAG \\
Xyr1-ver-R & CCTGGCAGCAATAAGAGAGC \\
Xyr1-ver-F & CCTTGCGATAAGTGGGATC \\
5Pxyr1-ver-F & CCAGCTGCCACTCTCATG \\
GFP-ver-R & AAGCACTGCACGCCGTA \\
GFP-ver-F & TACGGCGTGCAGTGCTC \\
Pgpdh-ver-F & TGCTAAGGTACCTAGGGAGGGA \\
hph-ver-R & CAAGCACTTCCGGAATCG \\
act-mp-F & ACTTCGGCCGCATTCTG \\
act-mp-R & AGCCAGGATCTTCATCAGGTAG \\
DNA sequencing & \\
Pxyr1-seq-F & \\
Txyr1-seq-F & GACAGCAGCAGTAGTCAGGT \\
xyr1-seq-F & TTGCTGAAAGTGAAGAGGG \\
5'xyr1-seq-F & CCGTCTCCCAAGACTAGC \\
3'xyr1-seq-F & CACATTGAGGCTCTGTC \\
Xyr1 IA2294-seq-F & AAGCATCTGCCATTGTCC \\
3'GFP-seq-F & GCCACGATGCAGGGAA \\
5'GFP-seq-F & CCGACAACCACTACCTGA \\
Pgpdh-seq-F & GCCACAAGTTCAGCGTG \\
Tgpdh-seq-R & CATGCAGTTGCTAAGGTACC \\
\hline
\end{tabular}

replacement experiments were performed using submerged germling cultures. For this, conidia from one week-old carbon source-free MA plate cultures were harvested in sterile water and cell concentration determined using a Thoma cell counting chamber. One hundred million cells were used to inoculate $100 \mathrm{ml}$ MA pre-culture medium (liquid MA medium with peptone to aid germination) in $500 \mathrm{ml}$ shake flasks, supplemented with either $50 \mathrm{mM}$ D-glucose (cellulase repressing condition) or $1 \%$ $\mathrm{w} / \mathrm{v}$ carboxymethyl-cellulose (cellulase inducing condition) as sole carbon source, and incubated at $28^{\circ} \mathrm{C}$ and $200 \mathrm{rpm}$ overnight in the dark. The next morning biomass from $20 \mathrm{ml}$ pre-culture aliquots were washed twice with sterile tap water and transferred into $20 \mathrm{ml}$ carbon source-free MA replacement medium (liquid MA medium w/o peptone) in $100 \mathrm{ml}$ shake flasks. If desired, germlings were starved for up to one hour under identical incubation conditions in order to drain internal carbon storage. At time point $\mathrm{t}=0 \mathrm{~min}$ a new carbon source for cellulase induction (1.5 mM sophorose, $1 \mathrm{mM}$ D-xylose, $25 \mathrm{mM}$ lactose or $1 \% \mathrm{w} / \mathrm{v}$ cellulose) was added and incubation continued. Cell samples for microscopical analysis were collected from overnight pre-cultures before and after carbon source replacement at desired time points. Carbon source-free MA cultures were used as controls for derepressing conditions.

Expression and subcellular localization of GFP-labeled fusion proteins was quantified using scanning confocal microscopy and image analysis as described in detail recently [19]. Briefly, average nuclear and cytoplasmic fluorescence intensities were calculated from at least 120 individual measurements per experimental condition (using fixed-size, circular measuring tools placed onto optically sectioned nuclei or into the adjacent cytoplasm) and graphically evaluated. Standard deviation bars in the respective graphs represent the considerable biological variation of transcription factor recruitment to the individual, mitotically and transcriptionally non-synchronized nuclei within the population, rather than a true statistical error.

Live-cell imaging was performed using a Nikon C1 confocal laser scanning unit mounted on a Nikon Eclipse TE2000-E inverted microscope base (Nikon $\mathrm{GmbH}$, Vienna, Austria). GFP-labelled proteins were excited with the $488 \mathrm{~nm}$ laser line of an argon ion laser, and the emitted fluorescence light separated by a Nikon MHX40500b/C100332 filter cube was detected with a photo-multiplier tube within the range of $500-530 \mathrm{~nm}$. A Nikon Plan Apo VC 60×/1.2 water immersion objective lens was used, and laser intensity and dwell time during image acquisition were kept to a minimum to reduce photobleaching and phototoxic effects. Brightfield images were captured simultaneously with a Nikon C1-TD transmitted light detector mounted behind the condenser turret. Images were recorded with a maximum resolution of $1024 \times 1024$ pixels and saved as TIFF. Apart from display range adjustments, images were not subjected to further manipulation. Intensity measurements were performed with the MacBiophotonics ImageJ work package available at (www.macbiophotonics.ca/ software.htm).

\section{Gene expression analysis}

RNA extraction was performed according to [60], and if necessary purified using the RNeasy MinElute Cleanup Kit (Qiagen, Hilden, Germany). RNA quality and quantity were determined using a Nanodrop spectrophotometer (Thermo Scientific, Vienna, Austria). Transcript quantification was performed by RT-qPCR. To this end, DNase I-treated (Fermentas, \#EN0521) RNA (3 $\mu \mathrm{g})$ was reverse-transcribed with the RevertAid First Strand cDNA Kit (Thermo Scientific, \#K1632) according to the manufacturer's protocol with a 1:1 combination of the provided oligo-dT and random hexamer primers.

All RT-qPCR experiments were performed on an Eppendorf realplex ${ }^{2}$ Mastercycler (Eppendorf, Hamburg, 
Germany). Each sample was prepared as $25 \mu$ l reaction using the iQ SYBR Green Supermix (Bio-Rad, \#1708882) with a final primer concentration of $100 \mathrm{nM}$ forward and reverse primer each. All assays were carried out as triplicates in a 96-well plate format covered with optical tape, including no-template controls. Measurements with the housekeeping gene translation elongation factor 1 (tef1) were performed for reference calculation and data normalization. Determination of the PCR efficiency was performed using triplicate reactions from a dilution series of cDNA $(1 ; 0.1 ; 0.01 ; 0.001)$. RT-qPCR primers, amplification efficiency and R-square values are given in Table 4. Amplification efficiency of each sample mRNA was then calculated from the given slopes in the iQ5 Optical system Software v2.0 and fold-changes in gene expression were calculated using REST Software [61]. All samples were analysed in at least two independent biological experiments with three RT-qPCR replicates in each run.

\section{Protein extraction, SDS-PAGE and western blot analysis} Overnight liquid pre-cultures (100 ml MA medium, 1\% w/v glucose) were prepared as described above for shuttling experiments. Samples for positive (QM6a; Ptef1:: $g f p$ ) and negative (QM9414 $\Delta t k u 70)$ controls were directly drawn from these pre-cultures after $18 \mathrm{~h}$ of incubation. Biosynthesis of GFP-XYR1 and GFP-XYR1 ${ }^{1-780}$, respectively, was induced by transferring fungal biomass from $50 \mathrm{ml}$ pre-culture into $50 \mathrm{ml}$ fresh MA medium containing $1.4 \mathrm{mM}$ sophorose. These induced cultures were harvested after 1,2 and/or 3 hours of additional incubation. For each sample, fungal biomass from $50 \mathrm{ml}$ liquid pre- or induced culture was harvested on a glass microfiber filter (Whatmann, cat.no. 1822-047) using vacuum-driven filtration, and, after washing twice with sterile tap water, immediately shock frozen in liquid nitrogen. Subsequently, the biomass was ground to a fine powder in liquid nitrogen, and approximately $100 \mathrm{mg}$

Table 4 RT-qPCR primers used in this study

\begin{tabular}{lllll}
\hline Gene & Primer name & $\mathbf{5}^{\prime}$-3' Sequence & $\mathbf{R}^{\mathbf{2}}$ & Efficiency \\
\hline cel7a & qPCR-cel7a-F & CCGAGCTGGTAGTTACTCTG & 0.990 & 0.98 \\
& qPCR-cel7a-R & GGTAGCCTTCTTGAACTGAGT & & \\
xyn2 & qPCR-xyn2-F & CAACCAGCCGTCCATCATCG & 0.993 & 0.97 \\
& qPCR-xyn2-R & ATCGTCCCGAGCGTCAGG & & \\
xyr1 & qPCR-xyr1-F & CCATCAACCTCTAGACGAC & 0.987 & 0.99 \\
& qPCR-xyr1-R & AACCCTGCAGGAGATAGAC & & \\
cre1 & qPCR-cre1-F & GTCTGAGAAACCTGTCCCTG & 0.996 & 0.91 \\
& qPCR-cre1-R & GGCTAATGATGTCGGTAAGTG & & \\
tef1 & qPCR-tef1-F & CCACATTGCCTGCAAGTTCGC & 0.995 & 0.95 \\
& qPCR-tef1-R & GTCGGTGAAAGCCTCAACGCA & & \\
\hline
\end{tabular}

of it were added to a $2 \mathrm{ml}$ Eppendorf tube already containing $1 \mathrm{ml}$ of protein extraction buffer $(10 \mathrm{ml}$ PBS containing $5 \mathrm{mM}$ EDTA and $5 \mathrm{mM}$ PMSF plus one cOmplete ULTRA protease inhibitor cocktail tablet (Roche, cat.no. 05892791 001), pH 7.4), and $1 \mathrm{~g}$ of small (0.25 mm diameter) and four large (3 mm diameter) glass beads to aid cell destruction. The mix was subjected to three oneminute rounds of homogenization at $30 \mathrm{~Hz}$ with oneminute cooling intervals at $-20^{\circ} \mathrm{C}$. Cell debris and aqueous phase were separated by centrifugation with $17.000 \times \mathrm{g}$ for $5 \mathrm{~min}$ and at $4^{\circ} \mathrm{C}$. The clear supernatant containing all soluble proteins was transferred into fresh, pre-cooled Eppendorf tubes and stored at $-20^{\circ} \mathrm{C}$ until further use. Total protein concentration was determined against BSA using Bradford reagent (BioRad, cat.no. 500-0006) according to manufacturer's recommendations. Typically, protein yields between $1-3 \mathrm{mg} / \mathrm{ml}$ were achieved.

Depending on the strain and protein of interest, e.g. for cytosolic GFP expressed under control of the constitutive $\mathrm{P}_{\text {tefi }}$ promoter in T. reesei QM6a much less total protein was required to yield a high detection signal on the Western blot, 5-30 $\mu \mathrm{g}$ of the crude total protein extract per lane were separated by SDS-PAGE as outlined in detail elsewhere [62]. Generally, two identical 12-14\% SDSPAGE gels were prepared, one for colloidal Coomassie staining, and the second for semi-dry electro blotting of the separated proteins onto ImmobileFL PVDF membrane (Millipore, cat.no. IPFL00010). Subsequent blocking was achieved by incubation in PBS-T (PBS, 0.3\% Tween 20) supplemented with $2 \%$ w/v milk powder (Roth, cat.no. T145.1) for 1 hour at room temperature. For the specific labelling of GFP and GFP-fusion proteins, respectively, the monoclonal mouse anti-GFP-HRP antibody $\alpha$-GFP(B-2) (Santa Cruz, cat.no. sc-9996) was used, diluted 1:1000 in PBS-T containing $0.5 \% \mathrm{w} / \mathrm{v}$ milk powder, and incubated on the membrane for 2 hours at room temperature, followed by four washing steps with PBS-T.

Detection of the labelled proteins was performed with the Pierce ECL2 kit (Thermo Scientific, cat.no. 80197) according to manufacturer's recommendations. Chemifluorescent signals were recorded on a Typhoon FLA700 imager (GE Healthcare), and chemiluminescent signals were visualized by $\mathrm{x}$-ray film (Amersham Hyperfilm ECL, GE Healthcare, cat.no. 28-9068-35) exposure. For protein band size estimation, two molecular weight markers were used: PageRuler Pre-stained Protein Ladder 10 - $170 \mathrm{kDa}$ (Thermo Scientific, cat.no. 26616), and SuperSignal Enhanced Protein Ladder 20 - 150 kDa (Thermo Scientific, cat.no. 84786), with only the latter one being applicable for ECL detection on x-ray film.

\section{Bioinformatic in silico analyses of XYR1}

Functional domains were analysed by Pfam domain search [15]. Coiled-coil regions were identified using 
COILS [16], and Protparam [18]) was used for the determination of general physicochemical properties of XYR1. The 3D-functional domain structure of XYR1 was determined by the RaptorX protein structure prediction software (raptorx.uchicago.edu; [17]).

\section{Additional files}

Additional file 1: Figure S1. Phenotypic comparison of QM6a and QM9136. Growth of T. reesei QM6a (parental strain) and QM9136 on solid minimal agar medium supplemented with either D-glucose, cellobiose or lactose.

Additional file 2: Table S1. Chromosomal location of mutated genes in QM9136.

Additional file 3: Figure S2. CDNA alignment of native and mutated xyr1 loci. CDNA alignment of the $5^{\prime}$-region of native xyr1 and mutated xyr1 ${ }^{\text {QM9136 }}$ loci showing the A2294 point deletion causing the frame-shift that introduces six stop codons; the first of which terminates translation pre-maturely leading to the truncated XYR $1^{1-780}$.

Additional file 4: Figure S3. Alignment of the C-terminus of XYR1 with the orthologs from several Pezizomycota. Abbreviations (=gene bank entries) specify: AAV31053.1, Penicillium canescens; AAZ75672.1, Sclerotinia sclerotiorum; aor:AO090012000267, Aspergillus oryzae; BAB62022.1, A. kawachii; EAA33375.1, Neurospora crassa; EAQ87362.1, Chaetomium globosum; XP_363488.1, Magnaporthe grisea; Xyr1_9136, T. reesei QM9136; XYR1_WT, T. reesei QM6a (parental strain).

Additional file 5: Figure S4. Detection of GFP-XYR1 under autofluorescence background eliminating conditions. To verify that the generally weak GFP-XYR1 ${ }^{1-780}$ fluorescence signals were no artifacts, microscope settings were optimized to eliminate cellular background auto-fluorescence, so that any detectable fluorescence signal must emanate from a GFP source. For this test we deliberately choose a weak inducer (1 mM D-xylose for $30 \mathrm{~min}$ ) to evaluate GFP-XYR1 ${ }^{1-780}$ fluorescence at the lower end of the protein expression and microscope detection range respectively. (A) The truncated GFP-XYR1 ${ }^{1-780}$ protein was detectable with an average intensity of about 4.5-times less compared to the full-length GFP-XYR1 construct. Interestingly, under these conditions GFP-XYR1 ${ }^{1-780}$ was localized as small cellular clusters (spots), and its cytoplasmic fraction was slightly elevated. This possibly suggests less efficient nuclear import due to proteasomal degradation of the non-functional construct. Notably, as shown above, in the presence of stronger cellulase-inducing carbon sources GFP-XYR1 ${ }^{1-780}$ does - although highly inefficient - accumulate inside nuclei. Nevertheless, in conclusion, this experiment confirmed that the truncated XYR1 allele becomes expressed and responds to cellulase-induction, however, its production within 30 minutes on $1 \mathrm{mM}$ D-xylose medium cannot be upregulated sufficiently, therefore leading to a low signal-to-noise ratio. (n.d. = not detectable). (B) Representative fluorescence and corresponding bright field images of the experimental conditions quantified in (A).

Additional file 6: Figure S5. Xyr 1 gene replacement cassette. Schematic representation of the replacement cassette for exchanging the native xyr1 locus in QM9136 and QM9414, respectively, with a gfp-xyr1 encoding fragment by homologous recombination. The approximate position of the A2294 point deletion inserted in the ffp-xyr $^{\triangle 42294}$ construct, leading to the expression of the truncated GFP-XYR1 $1^{1-780}$ protein, is indicated by an asterisk.

\section{Abbreviations}

AAD: Acidic activation domain; ATCC: American type culture collection; BLAST: Basic local alignment search tool; CBH1: Cellobiohydrolase 1; CDR: Coding region; DBD: DNA-binding domain; FSTFD: Fungal-specific transcription factor domain; FTFAD: Fungal transcription factor activation domain; FTFRMH: Fungal transcription factor regulating middle homology (domain); GAl: Genome analyser 1; GFP: Green fluorescent protein: indels: Insertions and deletions; JGI: Joint Genome Institute; MA: MandelsAndreotti; NADH: nicotinamid adenine dinucleotide (reduced form); NLS: Nuclear localization signal; PDA: Potato dextrose agar; QM: Quater master (strain collection); RUT: Rutgers (mutagenesis program); SNP: Short nucleotide polymorphism; SNV: Short nucleotide variants; SRA: Sequence read archive; TF: Transcription factor; TRAL: Trichoderma reesei Alexander Lichius; Trire: Trichoderma reesei; US: United States; WWII: Second world war; XYN2: Xylanase 2; XYR1: Xylanase regulator 1.

\section{Competing interests}

The authors declare that they have no competing interests.

\section{Authors' contributions}

AL designed and carried out the molecular genetic studies and drafted the manuscript. FBi designed and carried out genomic studies and helped to draft the manuscript. FBu and TA assisted in molecular genetic studies and helped to analyze data. SLeC, JM, WS, IVG, SEB, and AM sequenced and annotated the genome, and identified the mutations. BS and CPK planned the study, BS participated in its design and coordination, and helped to draft the manuscript. CPK performed the in silico protein analysis and drafted the manuscript. All authors read and approved the final manuscript.

\section{Acknowledgments}

Work in the Vienna Laboratory was supported by grants from the Austrian Science Fund P-23202 and I-1249 to CPK, and P24219 to BS. The work conducted by the U.S. Department of Energy Joint Genome Institute is supported by the Office of Science of the U.S. Department of Energy under Contract No. DE-AC02-05CH1 1231. The IBENS high-throughput sequencing facility is a member of the "France Génomique" consortium (ANR-10-INBS-0009). Work in IFPEN and ENS has been funded by the Tuck Foundation (Enerbio fund, Trisys project), as well as fellowship to FB).

The authors are grateful to Dr. Christa Ivanova for providing the T. reesei QM9414 tku70 strain, and the expert help of Dr. Lukas Hartl is also gratefully acknowledged.

\section{Author details}

'Research Division Biotechnology and Microbiology, Institute of Chemical Engineering, Vienna University of Technology, A-1060 Vienna, Austria. ${ }^{2}$ IFP Energies Nouvelles, 1-4 Avenue de Bois-Préau, 92852 Rueil-Malmaison, France. ${ }^{3}$ Sorbonne Universités, UPMC Université Paris 06, Institut de Biologie Paris Seine (IBPS), FR 3631, Département des Plateforme, F-75005 Paris, France. ${ }^{4}$ US Department of Energy, Joint Genome Institute, 2800 Mitchell Avenue, Walnut Creek, CA 94598, USA. ${ }^{5}$ Environmental Molecular Sciences Laboratory, Pacific Northwest National Laboratory, Richland, WA 99354, USA.

Received: 11 December 2014 Accepted: 13 April 2015

Published online: 20 April 2015

\section{References}

1. Liu G, Qin Y, Li Z, Qu Y. Development of highly efficient, low-cost lignocellulolytic enzyme systems in the post-genomic era. Biotechnol Adv. 2013;31:962-75.

2. Kubicek CP. Systems biological approaches towards understanding cellulase production by Trichoderma reesei. J Biotechnol. 2013;163:133-42.

3. Reese ET. History of the cellulase program at the U.S. army Natick Development Center. Biotechnol Bioeng Symp. 1976;6:9-20.

4. Bayer EA. The life and lifework of mary mandels - first lady of cellulase research. 2009.

5. Le Crom S, Schackwitz W, Pennacchio L, Magnuson JK, Culley DE, Collett JR, et al. Tracking the roots of cellulase hyperproduction by the fungus Trichoderma reesei using massively parallel DNA sequencing. Proc Natl Acad Sci. 2009;106:16151-6.

6. Vitikainen M, Arvas M, Pakula T, Oja M, Penttila M, Saloheimo M. Array comparative genomic hybridization analysis of Trichoderma reesei strains with enhanced cellulase production properties. BMC Genomics. 2010;11:441.

7. Porciuncula Jde O, Furukawa T, Mori K, Shida Y, Hirakawa H, Tashiro K, et al. Single nucleotide polymorphism analysis of a Trichoderma reese hyper-cellulolytic mutant developed in Japan. Biosci Biotechnol Biochem. 2013;77:534-43.

8. Koike H, Aerts A, LaButti K, Grigoriev IV, Baker SE. Comparative genomics analysis of trichoderma reesei strains. Industrial Biotechnology. 2013;9(6):352-67.

9. Mandels M, Weber J, Parizek R. Enhanced cellulase production by a mutant of Trichoderma viride. Appl Microbiol. 1971;21:152-4. 
10. Nevalainen KM, Palva ET. Production of extracellular enzymes in mutants isolated from Trichoderma viride unable to hydrolyze cellulose. Appl Environ Microbiol. 1978;35:11-6.

11. Torigoi E, Henrique-Silva F, Escobar-Vera J, Carle-Urioste JC, Crivellaro O, El-Dorry $\mathrm{H}$, et al. Mutants of Trichoderma reesei are defective in cellulose induction, but not basal expression of cellulase-encoding genes. Gene. 1996;173:199-203.

12. Stricker AR, Grosstessner-Hain K, Wurleitner E, Mach RL. Xyr1 (xylanase regulator 1) regulates both the hydrolytic enzyme system and D-xylose metabolism in Hypocrea jecorina. Eukaryotic Cell. 2006;5:2128-37.

13. Seidl V, Huemer B, Seiboth B, Kubicek CP. A complete survey of Trichoderma chitinases reveals three distinct subgroups of family 18 chitinases. FEBS J. 2005:272:5923-39.

14. Linder M, Mattinen ML, Kontteli M, Lindeberg G, Stahlberg J, Drakenberg T, et al. Identification of functionally important amino acids in the cellulose-binding domain of Trichoderma reesei cellobiohydrolase I. Protein Sci. 1995:4:1056-64.

15. Punta M, Coggill PC, Eberhardt RY, Mistry J, Tate J, Boursnell C, et al. The Pfam protein families database. Nucleic Acids Res. 2012;40:D290-301.

16. Lupas A, Van Dyke M, Stock J. Predicting coiled coils from protein sequences. Science. 1991;252:1162-4.

17. Kallberg M, Wang H, Wang S, Peng J, Wang Z, Lu H, et al. Template-based protein structure modeling using the RaptorX web server. Nat Protoc. 2012;7:1511-22.

18. Gasteiger E, Hoogland C, Gattiker A, Duvaud S, Wilkins MR, Appel RD, et al. Protein Identification and Analysis Tools on the ExPASy Server. In: Walker JM, editor. The Proteomics Protocols Handbook. Totowa, NJ: Humana Press Inc; 2005. p. 571-607

19. Lichius A, Seidl-Seiboth V, Seiboth B, Kubicek CP. Nucleo-cytoplasmic shuttling dynamics of the transcriptional regulators XYR1 and CRE1 under conditions of cellulase and xylanase gene expression in Trichoderma reesei. Mol Microbiol. 2014:94:1162-78.

20. Weld RJ, Plummer KM, Carpenter MA, Ridgway HJ. Approaches to functional genomics in filamentous fungi. Cell Research. 2006;16:31-44.

21. McCluskey K, Wiest AE, Grigoriev IV, Lipzen A, Martin J, Schackwitz W, et al. Rediscovery by whole genome sequencing: classical mutations and genome polymorphisms in Neurospora crassa. G3 (Bethesda). 2011;1:303-16.

22. Nowrousian M, Teichert I, Masloff S, Kuck U. Whole-Genome Sequencing of Sordaria macrospora Mutants Identifies Developmental Genes. G3 (Bethesda). 2012;2:261-70.

23. Irvine DV, Goto DB, Vaughn MW, Nakaseko Y, McCombie WR, Yanagida M, et al. Mapping epigenetic mutations in fission yeast using whole-genome next-generation sequencing. Genome Res. 2009;19:1077-83.

24. Pomraning KR, Smith KM, Freitag M. Bulk segregant analysis followed by high-throughput sequencing reveals the Neurospora cell cycle gene, ndc-1, to be allelic with the gene for ornithine decarboxylase, spe-1. Eukaryot Cell. 2011;10:724-33.

25. Schneeberger K, Ossowski S, Lanz C, Juul T, Petersen AH, Nielsen KL, et al. SHOREmap: simultaneous mapping and mutation identification by deep sequencing. Nat Methods. 2009;6:550-1.

26. Zuryn S, Le Gras S, Jamet K, Jarriault S. A strategy for direct mapping and identification of mutations by whole-genome sequencing. Genetics. 2010;186:427-30

27. Montenecourt BS, Eveleigh DE. Preparation of mutants of Trichoderma reesei with enhanced cellulase production. Appl Environ Microbiol. 1977;34:777-82.

28. Seiboth B, Herold S, Kubicek CP. Metabolic engineering of inducer formation for cellulase and hemicellulase gene expression in Trichoderma reesei. Sub-cellular Biochem. 2012;64:367-90

29. Tani S, Kawaguchi T, Kobayashi T. Complex regulation of hydrolytic enzyme genes for cellulosic biomass degradation in filamentous fungi. Appl Microbiol Biotechnol. 2014;98:4829-37.

30. Häkkinen M, Valkonen MJ, Westerholm-Parvinen A, Aro N, Arvas M, Vitikainen $\mathrm{M}$, et al. Screening of candidate regulators for cellulase and hemicellulase production in Trichoderma reesei and identification of a factor essential for cellulase production. Biotechnol Biofuels. 2014;7:14.

31. Seiboth B, Gamauf C, Pail M, Hartl L, Kubicek CP. The D-xylose reductase of Hypocrea jecorina is the major aldose reductase in pentose and D-galactose catabolism and necessary for beta-galactosidase and cellulase induction by lactose. Mol Microbiol. 2007:66:890-900.
32. MacPherson $S$, Larochelle $M$, Turcotte $B$. A fungal family of transcriptional regulators: the zinc cluster proteins. Microbiol Mol Biol Rev. 2006;70:583-604

33. Naar AM, Thakur JK. Nuclear receptor-like transcription factors in fungi. Genes Dev. 2009;23:419-32.

34. Schjerling P, Holmberg S. Comparative amino acid sequence analysis of the C6 zinc cluster family of transcriptional regulators. Nucleic Acids Res. 1996:24:4599-607.

35. Derntl C, Gudynaite-Savitch L, Calixte S, White T, Mach RL, Mach-Aigner AR. Mutation of the Xylanase regulator 1 causes a glucose blind hydrolase expressing phenotype in industrially used Trichoderma strains. Biotechnol Biofuels. 2013:6:62

36. van Peij NN, Gielkens MM, de Vries RP, Visser J, de Graaff LH. The transcriptional activator $X \ln R$ regulates both xylanolytic and endoglucanase gene expression in Aspergillus niger. Appl Environ Microbiol. 1998;64:3615-19.

37. van Peij NN, Visser J, de Graaff LH. Isolation and analysis of $x \operatorname{lnR}$, encoding a transcriptional activator co- ordinating xylanolytic expression in Aspergillus niger. Mol Microbiol. 1998;27:131-42.

38. Valsecchi I, Guittard-Crilat E, Maldiney R, Habricot Y, Lignon S, Lebrun R, et al. The intrinsically disordered C-terminal region of Arabidopsis thaliana TCP8 transcription factor acts both as a transactivation and self-assembly domain. Mol Biosyst. 2013;9:2282-95.

39. Rauscher R, Wurleitner E, Wacenovsky C, Aro N, Stricker AR, Zeilinger S, et al. Transcriptional regulation of xyn1, encoding xylanase I, in Hypocrea jecorina. Eukaryotic Cell. 2006;5:447-56.

40. Furukawa T, Shida Y, Kitagami N, Ota Y, Adachi M, Nakagawa S, et al. Identification of the cis-acting elements involved in regulation of xylanase II gene expression in Trichoderma reesei PC-3-7. Fungal Genet Biol. 2008:45:1094-102.

41. Shida Y, Furukawa T, Ogasawara W, Kato M, Kobayashi T, Okada H, et al. Functional analysis of the egl3 upstream region in filamentous fungus Trichoderma reesei. Appl Microbiol Biotechnol. 2008;78:515-24.

42. Hasper AA, Trindade LM, van der Veen D, van Ooyen AJ, de Graaff LH. Functional analysis of the transcriptional activator XInR from Aspergillus niger. Microbiology. 2004;150:1367-75

43. Mach RL, Strauss J, Zeilinger S, Schindler M, Kubicek CP. Carbon catabolite repression of xylanase I (xyn1) gene expression in Trichoderma reesei. Mol Microbiol. 1996;21:1273-81.

44. Zeilinger S, Schmoll M, Pail M, Mach RL, Kubicek CP. Nucleosome transactions on the Hypocrea jecorina (Trichoderma reesei) cellulase promoter cbh2 associated with cellulase induction. Mol Genet Genomics. 2003;270:46-55

45. Strauss J, Mach RL, Zeilinger S, Hartler G, Stoffler G, Wolschek M, et al. Cre1, the carbon catabolite repressor protein from Trichoderma reesei. FEBS Lett. 1995;376:103-7.

46. IImen M, Thrane C, Penttila M. The glucose repressor gene crel of Trichoderma: isolation and expression of a full-length and a truncated mutant form. Mol Gen Genet. 1996;251:451-60.

47. Portnoy T, Margeot A, Seidl-Seiboth V, Le Crom S, Ben Chaabane F, Linke R, et al. Differential regulation of the cellulase transcription factors XYR1, ACE2, and ACE1 in Trichoderma reesei strains producing high and low levels of cellulase. Eukaryotic Cell. 2011;10:262-71.

48. Zeilinger S, Haller M, Mach R, Kubicek CP. Molecular characterization of a cellulase-negative mutant of Hypocrea jecorina. Biochem Biophys Res Commun. 2000;277:581-8.

49. Kuhls K, Lieckfeldt E, Samuels GJ, Kovacs W, Meyer W, Petrini O, et al Molecular evidence that the asexual industrial fungus Trichoderma reesei is a clonal derivative of the ascomycete Hypocrea jecorina. Proc Natl Acad Sci U S A. 1996:93:7755-60.

50. Guangtao Z, Hartl L, Schuster A, Polak S, Schmoll M, Wang T, et al. Gene targeting in a nonhomologous end joining deficient Hypocrea jecorina. J Biotechnol. 2009;139:146-51.

51. Kubodera T, Yamashita N, Nishimura A. Transformation of Aspergillus sp. and Trichoderma reesei using the pyrithiamine resistance gene (ptrA) of Aspergillus oryzae. Biosci Biotechnol Biochem. 2002:66:404-6.

52. Mandels MM, Andreotti RE. The cellulose to cellulase fermentation. Proc Biochem. 1978:13:6-13.

53. Penttila $M$, Nevalainen $H$, Ratto $M$, Salminen $E$, Knowles J. A versatile transformation system for the cellulolytic filamentous fungus Trichoderma reesei. Gene. 1987;61:155-64. 
54. Sambrook J, Russell DW. Molecular cloning: a laboratory manual. 3rd ed. Cold Spring Harbor, New York: Cold Spring Harbor Laboratory Press; 2001.

55. Li H, Ruan J, Durbin R. Mapping short DNA sequencing reads and calling variants using mapping quality scores. Genome Res. 2008;18:1851-8.

56. Chen K, Wallis JW, McLellan MD, Larson DE, Kalicki JM, Pohl CS, et al. BreakDancer: an algorithm for high-resolution mapping of genomic structural variation. Nat Methods. 2009;6:677-81.

57. Hartl L, Kubicek CP, Seiboth B. Induction of the gal pathway and cellulase genes involves no transcriptional inducer function of the galactokinase in Hypocrea jecorina. J Biol Chem. 2007;282:18654-9.

58. Schuster A, Bruno KS, Collett JR, Baker SE, Seiboth B, Kubicek CP, et al. $A$ versatile toolkit for high throughput functional genomics with Trichoderma reesei. Biotechnol Biofuels. 2012;5:1.

59. Lichius A, Lord KM, Jeffree CE, Oborny R, Boonyarungsrit P, Read ND. Importance of MAP kinases during protoperithecial morphogenesis in Neurospora crassa. PLoS One. 2012;7:1-21.

60. Chirgwin JM, Przybyla AE, MacDonald RJ, Rutter WJ. Isolation of biologically active ribonucleic acid from sources enriched in ribonuclease. Biochemistry 1979;18:5294-9.

61. Pfaffl MW, Horgan GW, Dempfle L. Relative expression software tool (REST) for group-wise comparison and statistical analysis of relative expression results in real-time PCR. Nucleic Acids Res. 2002;30, e36.

62. Sambrook J, Russell DW. Molecular cloning: a laboratory manual. 2nd ed. Painview, NY: Cold Spring Harbor Lab. Press; 2001.

63. Marmorstein R, Harrison SC. Crystal structure of a PPR1-DNA complex: DNA recognition by proteins containing a Zn2Cys6 binuclear cluster. Genes Dev. 1994:8:2504-12.

\section{Submit your next manuscript to BioMed Central and take full advantage of:}

- Convenient online submission

- Thorough peer review

- No space constraints or color figure charges

- Immediate publication on acceptance

- Inclusion in PubMed, CAS, Scopus and Google Scholar

- Research which is freely available for redistribution 